\title{
INTEGRATION IN NONCOMMUTATIVE SYSTEMS $\left({ }^{1}\right)$
}

\section{BY}

\author{
FRANK M. STEWART
}

In this paper we introduce an abstract integral which includes both product integrals and additive integrals.

Thus our theory of Riemann integration embraces both the product integrals of Volterra [1, 2, 3] $\left(^{2}\right)$, Birkhoff [3], and Masani [1] and the classical additive integrals of Riemann [1] and Stieltjes [1]. Similarly our theory of Lebesgue integration includes the Lebesgue product integrals of Schlesinger [1] and Birkhoff [3] as well as the familiar Lebesgue integral [Lebesgue 1] and the additive integrals of Birkhoff [1] and Price [1].

This unified treatment is made possible by a suitable choice of the objects to be integrated-the "differentials" of Definition 3.15. We define integrals in a system with but a single operation. The use of only one operation simplifies appreciably the theory of product integration.

Certain new results on product integration appear as corollaries of our general theory. Thus, for functions with values in a normed ring, we prove the existence of the Stieltjes product integral of a continuous function with respect to a function of bounded variation. New theorems on the Lebesgue product integrability of Riemann product integrable functions are obtained. The existence theorem for Birkhoff's Riemann product integral in a nondistributive system is extended.

The paper is divided into four parts. In Part I, after some notational preliminaries, we introduce our concept of a differential and define certain properties of differentials. The other novelties in this part are two concepts that serve to replace convexity in studying integration in systems where convexity has no meaning. These are "stability of multiplication" (Definitions 3.21 and 3.22) and the use of subpartitions in defining integral ranges (Definitions 3.16-3.19(3).

Parts II and III are respectively studies of Riemann and Lebesgue integration in our system. The principal results are generalizations of (i) the theorem that an almost everywhere continuous function is Riemann integrable and (ii) the theorem that a Riemann integrable function is Lebesgue integrable.

Presented to the Society, February 28, 1948; received by the editors April 16, 1949.

(1) The author wishes to acknowledge the help and encouragement he has received from Professors Garrett Birkhoff, Saunders MacLane, and Marshall Stone.

(2) Numbers in brackets refer to the bibliography at the end of this paper.

( ${ }^{3}$ In this connection, see the summary of a lecture by G. B. Price on pp. 176-177 of volume 48 of Bull. Amer. Math. Soc. 
In Part IV we consider the relation of our integral to some other integrals.

\section{Part I. Preliminaries}

1. Partitions. By a simple Riemann partition, or $S-P$, of an interval( $\left.{ }^{4}\right)$ $[\alpha, \beta]$ we mean a representation of $[\alpha, \beta]$ as a union of a finite number of disjoint intervals (some of which may consist of a single point). The S-P of $[\alpha, \beta]$ as $\left(^{5}\right) \bigcup_{i=1}^{n} \Delta_{i}$ will be denoted by $\Pi: \Delta_{i} ; i=1,2, \cdots, n$ or $\Pi: \Delta_{i}$ or simply II. The $\Delta_{i}$ of an S-P will always be indexed according to their natural ordering in the interval. The length of the interval $\Delta$ will be denoted by $|\Delta|$ and the norm of an S-P, $\Pi: \Delta_{i}$, by $|\Pi|=\sup _{i=1, \cdots, n}\left|\Delta_{i}\right|$.

An S-P, $\Pi: \Delta_{i}$, is a refinement of an S-P, $\Pi^{\prime}: \Delta_{j}^{\prime}$ (written $\Pi<\Pi^{\prime}$ ) if each $\Delta_{i}$ is contained in some $\Delta_{j}^{\prime}$. If $\Pi: \Delta_{i}, \Pi^{\prime}: \Delta_{j}^{\prime}$ are S-P's of an interval $[\alpha, \beta]$, then $\Pi \cdot \Pi^{\prime}$ stands for the S-P whose intervals are all nonvoid sets of the form $\Delta_{i} \cap \Delta_{j}^{\prime}$. Clearly $\Pi \cdot \Pi^{\prime}$ is characterized by the properties $\Pi \cdot \Pi^{\prime}<\Pi, \Pi \cdot \Pi^{\prime} \prec \Pi^{\prime}$, and $\Pi^{*}<\Pi$ and $\Pi^{*} \prec \Pi^{\prime}$ imply $\Pi^{*}<\Pi \cdot \Pi^{\prime}$.

By a double Lebesgue partition, or $L-P$, of $[\alpha, \beta]$ we mean a class of representations of $[\alpha, \beta]$ as $\bigcup_{i=1}^{n} \bigcup_{a \in A_{i}} \sigma_{i}^{a}$ where the $\sigma_{i}^{a}$ are disjoint, Lebesgue measurable subsets of $[\alpha, \beta]$ such that $\Pi: \Delta_{i}=\bigcup_{a \in A_{i}} \sigma_{i}^{a}$ is an S-P of $[\alpha, \beta]$, and the $A_{i}$ are linearly ordered countable sets. Two such representations $\bigcup_{i=1}^{n} \bigcup_{a \in A_{i}} \sigma_{i}^{a}$ and $\bigcup_{j=1}^{m} \bigcup_{b \in B_{i}} \sigma_{j}^{\prime b}$ belong to the same L-P if $m=n$ and there is a 1-to-1 correspondence (not necessarily order preserving) $a \leftrightarrow f_{i}(a) \in B_{i}$ between $A_{i}$ and $B_{i}$ such that $\sigma_{i}^{a}=\sigma_{i}^{\prime f(a)}$. The L-P, one of whose members is $\bigcup_{i=1}^{n} \bigcup_{a \in A_{i}} \sigma_{i}^{a}$, will be denoted by $L: \sigma_{i}^{a} ; i=1, \cdots, n ; a \in A_{i}$, or $L: \sigma_{i}^{a}$ or $L$. If $L: \sigma_{i}^{a}$ is an L-P, $\Pi(L)$ stands for the S-P, $\Pi: \bigcup_{a \in A_{i}} \sigma_{i}^{a}$. The Lebesgue measure of a measurable set, $\sigma$, will be denoted by $|\sigma|$.

An L-P, $L: \sigma_{i}^{a}$ is a refinement of the L-P, $L^{\prime}: \sigma_{j}^{\prime b}$ if $\Pi(L) \prec \Pi\left(L^{\prime}\right)$ and if each $\sigma_{i}^{a}$ is contained in some $\sigma_{j}^{\prime b}$. No confusion results from using $\prec$ for "is a refinement of" between L-P's as well as between S-P's. Clearly:

(1.1) The relation $\prec$ between L-P's is transitive.

(1.2) There is a unique L-P, $L \cdot L^{\prime}$, characterized by the properties $L \cdot L^{\prime}$ $\prec L, L \cdot L^{\prime} \prec L^{\prime}$, and $L^{*} \prec L, L^{*} \prec L^{\prime}$ imply $L^{*} \prec L \cdot L^{\prime}$.

A double Riemann partition or $R-P$ is an L-P, $R: s_{i}^{a} ; i=1, \cdots, n ; a \in A_{i}$ such that each $s_{i}^{a}$ is a union of a finite number of intervals and each $A_{i}$ is finite.

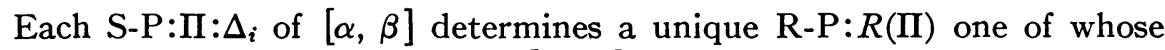
members is the representation( $\left.{ }^{6}\right)[\alpha, \beta]=\bigcup_{i=1}^{n} \bigcup_{a \in\{1\}} \Delta_{i}^{a}$ where $\Delta_{i}^{1}=\Delta_{i}$. In general we shall not distinguish between $\Pi$ and $R(I I)$.

$\left.{ }^{4}\right)$ The symbols $[\alpha, \beta],(\alpha, \beta)$ stand respectively for the closed and open intervals with end points $\alpha$ and $\beta$. Half open intervals are denoted by $[\alpha, \beta)$ and $(\alpha, \beta]$.

(5) The symbols $\subset, \cup, \cap$ denote, respectively, set theoretic "included in," "union," and "intersection." They will be used together with their usual variations. $A-B$ denotes the set of elements in $A$ but not in $B . x \in A$ means $x$ is an element of $A$.

(b) We use $\left\{a_{1}, a_{2}, \cdots, a_{n}\right\}$ for the set whose elements are $a_{1}, \cdots, a_{n}$. 
2. Limits. The limits we shall use are based on the idea of Moore-Smith convergence of sets (E. H. Moorè and H. L. Smith [1], Birkhoff [2]).

Observe:

(2.1) The S-P's of an interval $[\alpha, \beta]$ are directed by each of the relations $\prec$ and $\leqq$.

(2.2) The R-P's, also the L-P's, of an interval $[\alpha, \beta]$ are directed by the relation $\prec$.

Let sets $A(\Pi), B(R), C(L)$ in a Hausdorff space, $\mathscr{D}$, be defined respectively for each S-P, $\Pi$, each R-P, $R$, and each L-P, $L$ of an interval $[\alpha, \beta]$.

(2.3) Definition. Let $\lim _{|\mathrm{m}| \rightarrow 0} A(\Pi)=x$ mean: for any neighborhood $U_{x}$ of $x$ there is a $\delta>0$ such that $|\Pi|<\delta$ implies $A(\Pi) \subset U_{x}$.

(2.4) Definition. Let $\lim _{\Pi \downarrow} A(\Pi)=x$ mean: for any neighborhood $U_{x}$ of $x$ there is an S-P, $\Pi$, such that $\Pi^{\prime}<\Pi$ implies $A\left(\Pi^{\prime}\right) \subset U_{x}$.

(2.5) Definition. Let $\lim _{R \downarrow} B(R)=x$ mean: for any neighborhood $U_{x}$ of $x$ there is an R-P, $R$, such that $R^{\prime} \prec R$ implies $B\left(R^{\prime}\right) \subset U_{x}$.

(2.6) Definition. Let $\lim _{L \downarrow} C(L)=x$ mean: for any neighborhood $U_{x}$ of $x$ there is an L-P, $L$, such that $L^{\prime} \prec L$ implies $C\left(L^{\prime}\right) \subset U_{x}$.

The following is an immediate consequence of a result of Moore and Smith [1, pp. 106-107]( $\left.{ }^{7}\right)$.

(2.7) If $\mathfrak{D}$ is a complete metric space and if for any S-P's $\Pi_{1}$ and $\Pi_{2}$ of $[\alpha, \beta]$ the sets $A\left(\Pi_{1}\right)$ and $A\left(\Pi_{2}\right)$ have a point in common, then a necessary and sufficient condition that $\lim _{\Pi \Pi \rightarrow \rightarrow 0} A(\Pi)$ exist is that given $\epsilon>0$ there is a $\delta>0$ such that $|\Pi|<\delta$ implies $\left(^{8}\right) d(A(\Pi))<\epsilon$. Similar results obtain for $\lim _{\Pi \downarrow} A(\Pi)$, $\lim _{R \downarrow} B(R)$, and $\lim _{L \downarrow} C(L)$. Since $\Pi^{\prime} \prec \Pi$ implies $\left|\Pi^{\prime}\right| \leqq|\Pi|$, we have:

(2.8) If $\lim _{|\Pi| \rightarrow 0} A(\Pi)=x$, then $\lim _{\Pi} \downarrow A(\Pi)=x$.

(2.9) Definition. If an element, $f(\sigma)$ of a topological space, is defined for $\sigma$ an element of a class, $\subseteq$, of subsets of a topological space, then $\lim _{\sigma \rightarrow t} f(\sigma)=x$ means given any neighborhood $U_{x}$ of $x$, then there is a neighborhood $V_{t}$ of $t$ such that $\sigma \subset V_{t}$ and $\sigma \in \subseteq$ imply $f(\sigma) \in U_{x}$.

3. Abstract differentials. As a background for our introduction of abstract differentials and integrals we restate some familiar definitions in a notation similar to that we shall employ for their abstract counterparts.

Let $f$ stand for a real-valued function with domain $[\alpha, \beta]$ and let $\Pi, R$, and $L$ stand respectively for an S-P, an R-P, and an L-P of $[\alpha, \beta]$. Let:

(3.1) $J\left(f, \Pi: \Delta_{i}\right)$ be the set of all sums of the form $\sum_{i=1}^{n} f\left(t_{i}\right) \cdot\left|\Delta_{i}\right|$ with $t_{i} \in \Delta_{i}$;

(3.2) $S J(f, \Pi)$ be the set of all sums of the form $\sum_{j=1}^{n^{\prime}} f\left(t_{j}^{\prime}\right) \cdot\left|\Delta_{j}^{\prime}\right|$ where $t_{j}^{\prime} \in \Delta_{j}^{\prime}$ and $\Pi^{\prime}: \Delta_{j}^{\prime}<\Pi$;

(3.3) $R J(f, R)$ be the set of all sums of the form $\sum_{j=1}^{n} \sum_{b \in B_{i}} f\left(t_{j}^{\prime b}\right) \cdot\left|s_{j}^{\prime b}\right|$ where $t_{j}^{\prime} \in s_{j}^{\prime b}$ and $R^{\prime}: s_{j}^{\prime b}<R$;

${ }^{(7)}$ Their result is for the case where $\mathfrak{D}$ is the real number system, but the proof is readily extended to the case where $\mathfrak{D}$ is any complete metric space.

${ }^{(8)}$ The diameter of a set $A$ in a metric space will be denoted by $d(A)$ throughout this paper. 
(3.4) $L J(f, L)$ be the set of all sums of the form $\sum_{j=1}^{n \prime} \sum_{b \in B_{i}} f\left(t_{j}^{\prime b}\right) \cdot\left|\sigma_{j}^{\prime b}\right|$ where $t_{j}^{\prime b} \in \sigma_{j}^{\prime b}$ and $L^{\prime}: \sigma_{j}^{\prime b}<L$.

The following statements are equivalent:

(3.5) $\lim _{|\Pi| \rightarrow 0} J(f, \Pi)=l$;

(3.6) $\lim _{\Pi \downarrow} J(f, \Pi)=l$;

(3.7) $\lim _{|\Pi| \rightarrow 0} S J(f, \Pi)=l$;

(3.8) $\lim _{\Pi \downarrow} S J(f, \Pi)=l$;

(3.9) $\lim _{R \downarrow} R J(f, R)=l$.

The common limit, if it exists, is the ordinary Riemann integral of $f$ over $[\alpha, \beta]$, while the Lebesgue integral of $f$ is $\lim _{L \downarrow} L J(f, L)$.

We are primarily interested in the abstract counterparts of $R J(f, R)$ and $L J(f, L)$. The counterparts of $J(f, \Pi)$ and $S J(f, \Pi)$ are used to make connections with known theories.

Let $F$ be a function with domain $[\alpha, \beta]$ and whose values are $n \times n$ matrices.

(3.10) Let $J\left(F, \Pi: \Delta_{i}\right)$ be the set of all products of the form $\prod_{i=1}^{n}\left(I+F\left(t_{i}\right)\right.$ $\left.\cdot\left|\Delta_{i}\right|\right)$ with $t_{i} \in \Delta_{i}$.

The product integral $\int_{\alpha}^{\beta}[I+F d t]$ of Volterra $\left[2\right.$, p. 35] is $\lim _{|\Pi| \rightarrow 0} J(F, \Pi)$.

Consider the common features of these situations. In (3.1-3.4) and in (3.10) we use a binary associative operation to combine objects (numbers or matrices) determined by a point of $[\alpha, \beta]$ and a subset of $[\alpha, \beta]$. A topology is needed so that we may take the limits which are the integrals. Unnecessary for definitions, but important for the development to follow, is the existence of an identity with respect to the binary operation used.

With these models in mind we adopt the following conventions.

(3.11) $\mathfrak{D}$ is a set of elements, $x, y, e, \cdots$.

(3.12) There is a binary associative operation . (multiplication) on $\mathfrak{D D}$ to $\mathfrak{D}$. The symbols $x_{1} \cdot x_{2} \cdots \cdots x_{n}, \prod_{i=1}^{n} x_{i}$, and $\prod_{a \in A} x_{a}$ where $A$ is a linearly ordered set are to have their obvious meanings in terms of $\cdot$

(3.13) There is an $e \in \mathfrak{D}$ such that $e \cdot x=x \cdot e=x$ for all $x \in \mathfrak{D}$.

(3.14) There is a Hausdorff topology on $\mathfrak{D}$. Except briefly in $\S 11$ it is to be understood that this topology is given by a specific metric $\rho$ and that $\mathfrak{D}$ is complete in this metric.

(3.15) Definition. A differential on an interval $[\alpha, \beta]$ is a function, $\mu$, of two variables such that $\mu\{t, \sigma\} \in \mathcal{D}$ whenever $t \in[\alpha, \beta]$ and $\sigma$ is a Lebesgue measurable subset of $[\alpha, \beta]$.

For the study of Riemann integration it would suffice to consider as differentials functions, $\mu$, such that $\mu\{t, s\} \in \mathfrak{D}$ whenever $t \in[\alpha, \beta]$ and $s$ is a union of a finite number of intervals contained in $[\alpha, \beta]$.

(3.16) Definition. The ordinary integral range of a differential, $\mu$, on $[\alpha, \beta]$ relative to the $\mathrm{S}-\mathrm{P}, \Pi: \Delta_{i}$, of $[\alpha, \beta]$ is the set, $J(\mu, \Pi)$, of all products of the form $\prod_{i=1}^{n} \mu\left\{t_{i}, \Delta_{i}\right\}$ where $t_{i} \in \Delta_{i}$.

(3.17) Definition. The simple integral range of a differential, $\mu$, on $[\alpha, \beta]$ relative to the $S-P, \Pi$, of $[\alpha, \beta]$ is the set $S J(\mu, \Pi)$ of all products of 
the form $\prod_{j=1}^{n^{\prime}} \mu\left\{t_{j}^{\prime}, \Delta_{j}^{\prime}\right\}$ where $\Pi^{\prime}: \Delta_{j}^{\prime}<\Pi$ and $t_{j}^{\prime} \in \Delta_{j}^{\prime}$.

(3.18) Definition. The Riemann integral range of a differential, $\mu$, on $[\alpha, \beta]$ relative to the $\mathrm{R}-\mathrm{P}, R$, of $[\alpha, \beta]$ is the set, $R J(\mu, R)$, of all products of the form $\prod_{j=1}^{n^{\prime}} \prod_{k=1}^{n_{j}^{\prime}} \mu\left\{t_{j}^{\prime k}, s_{j}^{\prime k}\right\}$ where $R^{\prime}: s_{j}^{\prime k}<R$.

(3.19) Definition. The Lebesgue integral range of a differential, $\mu$, on $[\alpha, \beta]$ relative to the $\mathrm{L}-\mathrm{P}, L$, of $[\alpha, \beta]$ is the set, $L J(\mu, L)$, of all products of the form $\prod_{j=1}^{n^{\prime}} \prod_{b \in B_{j}} \mu\left\{t_{j}^{\prime b}, \sigma_{j}^{\prime b}\right\}$ where $L^{\prime}: \sigma_{j}^{\prime b} \prec L$.

The discussion of the meaning of the infinite products occurring in Definition 3.19 will be deferred to $\S 9$. If some of them do not converge, $L J(\mu, L)$ is to be undefined.

(3.20) Definition. The ordinary integral, simple integral, Riemann integral, and Lebesgue integral of a differential, $\mu$, on $[\alpha, \beta]$ from $\alpha$ to $\beta$ are respectively

$$
\begin{aligned}
\int_{\alpha}^{\beta} \mu\{t, d t\} & =\lim _{|\Pi| \rightarrow 0} J(\mu, \Pi), \\
(S) \int_{\alpha}^{\beta} \mu\{t, d t\} & =\lim _{|\Pi| \rightarrow 0} S J(\mu, \Pi), \\
(R) \int_{\alpha}^{\beta} \mu\{t, d t\} & =\lim _{|\Pi| \rightarrow 0} R J(\mu, \Pi), \\
\text { (L) } \int_{\alpha}^{\beta} \mu\{t, d t\} & =\lim _{L \downarrow} L J(\mu, L) .
\end{aligned}
$$

Some strong assumption about the continuity of multiplication is required for our work. The following may be regarded as substitutes for the concept of the convexity of a linear space which plays an important role in additive integration theory.

(3.21) Definition. Multiplication in $\mathfrak{D}$ is absolutely stable (briefly $\mathfrak{D}$ is absolutely stable) if given $\epsilon>0$ there is a $\delta(\epsilon)>0$ such that $\sum_{i=1}^{n} \rho\left(x_{i}, y_{i}\right)$ $<\delta(\epsilon)$ implies $\rho\left(\prod_{i=1}^{n} x_{i}, \prod_{i=1}^{n} y_{i}\right)<\epsilon$.

(3.22) Definition. Multiplication in $\mathfrak{D}$ is relatively stable (briefly $\mathfrak{D}$ is relatively stable) if given $L>0, \epsilon>0$ there is a $\delta_{L}(\epsilon)>0$ such that $\sum_{i=1}^{n} \rho\left(x_{i}, e\right)$ $\leqq L$ and $\sum_{i=1}^{n} \rho\left(x_{i}, y_{i}\right)<\delta_{L}(\epsilon)$ imply $\rho\left(\prod_{1=1}^{n} x_{i}, \prod_{i=1}^{n} y_{i}\right)<\epsilon$.

As a consequence of the convexity of the norm we have:

(3.23) Addition in a Banach space is absolutely stable.

From an inequality given by Masani $[1$, p. 153, equation (4.43)] we infer:

(3.24) Multiplication in a normed ring is relatively stable.

4. Properties of differentials. If we consider the case where $\mathfrak{D}$ is the set of real numbers with the operation of addition and the usual metric and if $\mu\{t, \sigma\}=f(t) \cdot|\sigma|$, the statement $f$ is bounded by $B$ is equivalent to $\rho(\mu\{t, \sigma\}, 0) \leqq B \cdot|\sigma|$. On the other hand, if $\mu\{t, s\}=f(t) \sum_{i=1}^{j}\left[u\left(\bar{t}_{i}\right)-u\left(t_{i}^{\prime}\right)\right]$ whenever $s$ is the union of disjoint intervals $\Delta_{i}, i=1, \cdots, j$, with end points 
$t_{i}^{\prime}$ and $\bar{t}_{i}$, the boundedness of $f$ does not imply $\rho(\mu\{t, s\}, 0) \leqq B \cdot|s|$. (Such differentials lead to Stieltjes integrals.) However if $f$ is bounded and $u$ is of bounded variation on $[\alpha, \beta]$, then the sums $\sum_{i=1}^{n} \sum_{j=1}^{n_{i}} \rho\left(\mu\left\{t_{i}^{j}, s_{i}^{j}\right\}, 0\right)$ are uniformly bounded for all R-P's, $R: \mathrm{s}_{i}^{j}$ of $[\alpha, \beta]$. These observations suggest the following definitions.

(4.1) Definition. A differential on $[\alpha, \beta], \mu$, is bounded if for some $B$ and all measurable sets $\sigma \subset[\alpha, \beta] \rho(\mu\{t, \sigma\}, e) \leqq B \cdot|\sigma|$. Any such $B$ is a bound of $\mu$.

(4.2) Definition. A differential on $[\alpha, \beta], \mu$, is of bounded $R$-variation if there is a number $V$ such that $\sum_{i=1}^{n} \sum_{j=1}^{n_{i}} \rho\left(\mu\left\{t_{i}^{j}, s_{i}^{j}\right\}, e\right) \leqq V$ for all R-P's, $R: s_{i}^{j}$, of $[\alpha, \beta]$ and all $t_{i}^{j} \in s_{i}^{j}$.

Clearly:

(4.3) If a differential on $[\alpha, \beta]$ is bounded it is of bounded $R$-variation.

Returning to the case where $\mu\{t, \sigma\}=f(t) \cdot|\sigma|$ where $f$ is a real valued function, we observe that the oscillation of $f$ on an interval $\Delta$ may be written in terms of $\mu$ as

$$
\sup _{t_{1} \in \Delta, t_{2} \in \Delta,|\sigma|>0} \frac{\rho\left(\mu\left\{t_{i}, \sigma\right\}, \mu\left\{t_{2}, \sigma\right\}\right)}{|\sigma|} .
$$

For an abstract differential $\rho\left(\mu\left\{t_{1}, \sigma\right\}, \mu\left\{t_{2}, \sigma\right\}\right) /|\sigma|$ may depend on $\rho$ and it is natural to take the supremum only over sets $\sigma \subset \Delta$. In establishing the existence of Riemann-Stieltjes product integrals in a normed ring (cf. \$12) we require a generalization of this idea embodied in the following definitions.

(4.4) Definition. If $m$ is a non-negative additive set function whose domain includes all finite unions of subintervals of $[\alpha, \beta]$ and if $\mu$ is a differential on $[\alpha, \beta]$, then the $m$-oscillation of $\mu$ on an interval $\Delta \subset[\alpha, \beta]$ with $m(\Delta)>0$ is

$$
\operatorname{Osc}_{m}(\mu \mid \Delta)=\sup _{t_{1}, t_{2}, s} \frac{\rho\left(\mu\left\{t_{1}, s\right\}, \mu\left\{t_{2}, s\right\}\right)}{m(s)}
$$

where $t_{1}, t_{2}$, and $s$ are restricted as follows: $t_{1}, t_{2} \in \Delta, s$ is a union of a finite number of intervals, $s \subset \Delta$, and $m(s)>0$. If $m(\Delta)=0$, we define $\operatorname{Osc}_{m}(\mu \mid \Delta)$ as 0 if $\rho\left(\mu\left\{t_{1}, s\right\}, \mu\left\{t_{2}, s\right\}\right)=0$ for all $t_{1}, t_{2} \in \Delta$ and $s \subset \Delta$ and as $+\infty$ otherwise. If $m$ is Lebesgue measure, we write Osc $(\mu \mid \Delta)$ for $\operatorname{Osc}_{m}(\mu \mid \Delta)$.

(4.5) Definition. The $m$-oscillation of a differential on $[\alpha, \beta], \mu$, at $t \in[\alpha, \beta]$ is

$$
\operatorname{Osc}_{m}(\mu \mid t)=\underset{\Delta \rightarrow t}{\limsup } \operatorname{Osc}_{m}(\mu \mid \Delta) \text {. }
$$

We write Osc $(\mu \mid t)$ for $\operatorname{Osc}_{m}(\mu \mid t)$ if $m$ is Lebesgue measure.

Definitions (4.2) and (4.5), though motivated by consideration of differentials which lead to additive integrals, have simple interpretations if $\mathfrak{D}$ is a normed ring with the operation of multiplication and $\mu\{t, \sigma\}=I+F(t)$ $\cdot|\sigma|$. In this case: 
(4.6) The function $F$ is bounded if and only if $\mu$ is bounded.

(4.7) The function $F$ is continuous at $t$ if and only if $\operatorname{Osc}(\mu \mid t)=0$.

(4.8) Definition. A differential, $\mu$, on $[\alpha, \beta]$ is approximately multiplicative if there is a null function $\left(^{9}\right) \eta$ such that if $\sigma_{i}, i=1,2, \cdots, n$, are disjoint measurable subsets of $[\alpha, \beta]$, then

$$
\rho\left(\prod_{i=1}^{n} \mu\left\{t, \sigma_{i}\right\}, \mu\left\{t, \bigcup_{i=1}^{n} \sigma_{i}\right\}\right)<\eta\left(\left|\bigcup_{i=1}^{n} \sigma_{i}\right|\right) \cdot\left|\bigcup_{i=1}^{n} \sigma_{i}\right| .
$$

The significance of Definition 4.8 may be clarified by calculating explicitly the left-hand side of (4.9) in case $\mu\{t, \sigma\}=f(t) \cdot|\sigma|$ and $\mathfrak{D}$ is the real numbers and also in case $\mathfrak{D}$ is a normed ring with the operation of multiplication and $\mu\{t, \sigma\}=I+F(t) \cdot|\sigma|$.

\section{PART II. RIEMANN INTEgRation}

5. Relations between the integrals. Important consequences of Definitions 3.16-3.19 are

(5.1) $J(\mu, \Pi) \subset S J(\mu, \Pi) \subset R J(\mu, \Pi)$, and these are contained in $L J(\mu, \Pi)$ if it exists.

(5.2) If $\Pi^{\prime} \prec \Pi$, then $S J\left(\mu, \Pi^{\prime}\right) \subset S J(\mu, \Pi)$.

(5.3) If $R^{\prime}<R$, then $R J\left(\mu, R^{\prime}\right) \subset R J(\mu, R)$.

(5.4) If $L^{\prime}<L$, then $L J\left(\mu, L^{\prime}\right) \subset L J(\mu, L)$.

It is 5.4 that enables us to dispense with the use of convex hulls in discussing Lebesgue integrals. In fact if $\mathfrak{D}$ is a Banach space and $\mu\{t, \sigma\}=T(t)$ $\cdot|\sigma|$, the closure of $L J(\mu, L)$ is Birkhoff's [1, p. 367, Definition 2] integral range $J_{L}(T)$ and our 5.4 is essentially Birkhoff's Theorem 11 .

From 5.2 we infer that $S J\left(\mu, \Pi_{1} \cdot \Pi_{2}\right) \subset S J\left(\mu, \Pi_{1}\right) \cap S J\left(\mu, \Pi_{2}\right)$ for any S-P's $\Pi_{1}$ and $\Pi_{2}$. Hence from 2.7 we obtain:

(5.5) THEOREM. If $\mu$ is a differential on $[\alpha, \beta]$, a necessary and sufficient condition that $(S) \int_{\alpha}^{\beta} \mu\{t, d t\}$ exist is that given $\epsilon>0$ there is a $\delta>0$ such that $|\Pi|<\delta$ implies $d(S J(\mu, \Pi))<\epsilon$.

(5.6) THEOREM. If $\mu$ is a differential on $[\alpha, \beta]$, a necessary and sufficient condition that $\lim _{\Pi \downarrow} S J(\mu, \Pi)$ exist is that given $\epsilon>0$ there is an $\mathrm{S}-\mathrm{P}, \Pi(\epsilon)$, of $[\alpha, \beta]$ such that $\Pi<\Pi(\epsilon)$ implies $d(S J(\mu, \Pi))<\epsilon$.

Using (5.3) instead of (5.2) we obtain:

(5.7) Theorem. Theorems 5.5 and 5.6 remain valid if " $(S) \int_{\alpha}^{\beta} \mu\{t, d t\}$ " and "SJ( $\mu, \Pi)$ " are replaced by “ $(R) \int_{\alpha}^{\beta} \mu\{t, d t\}$ " and " $R J(\mu, \Pi)$ " respectively.

$\left({ }^{9}\right)$ We use the term null function for a real-valued function $f$ whose domain includes the non-negative real numbers and such that $f(0)=\operatorname{Lim}_{r \rightarrow 0+} f(r)=0$. Without loss of generality, the null function of Definition 4.8 will be taken to be nondecreasing. An equivalent $\epsilon-\delta$ definition seems to be less convenient in the applications. 
(5.8) THEOREM. If $\mathfrak{D}$ is relatively stable and $\mu$ is a bounded differential on $[\alpha, \beta]$, then the following statements are equivalent:

$$
\begin{gathered}
\int_{\alpha}^{\beta} \mu\{t, d t\}=\lim _{|\Pi| \rightarrow 0} J(\mu, \Pi)=x, \\
(S) \int_{\alpha}^{\beta} \mu\{t, d t\}=\lim _{|\Pi| \rightarrow 0} S J(\mu, \Pi)=x, \\
\lim _{\Pi \downarrow} J(\mu, \Pi)=x, \\
\lim _{\Pi \downarrow} S J(\mu, \Pi)=x .
\end{gathered}
$$

Proof. That (5.9) and (5.10) are equivalent as are (5.11) and (5.12) follows at once from Definitions (2.3), (2.4), (3.16), (3.17), and (3.20). From (2.8) and the above we see that either (5.9) or (5.10) implies (5.11) and (5.12). These implications hold whether $\mu$ is bounded or not.

Now assume (5.11) obtains. From Definition 2.4 we know that for any $\epsilon>0$ there is an S-P, $\Pi: \Delta_{i}, i=1,2, \cdots, n$, such that $\Pi^{\prime}<\Pi$ and $y \in J\left(\mu, \Pi^{\prime}\right)$ imply $\rho(y, x)<\epsilon$. Let $\Pi^{\prime \prime}: \Delta_{j}^{\prime \prime}, j=1,2, \cdots, n^{\prime \prime}$ be any S-P and $z=\prod_{j=1}^{n^{\prime \prime}}$ $\mu\left\{t_{j}^{\prime \prime}, \Delta_{j}^{\prime \prime}\right\}$ any element of $J\left(\mu, \Pi^{\prime \prime}\right)$. We procede to calculate an upper bound for $\rho(x, z)$.

Let the $t_{j}^{\prime \prime}$ and the $\Delta_{j}^{\prime \prime}$ be reindexed as $t_{i}^{\prime \prime k}, \Delta_{i}^{\prime \prime k}, i=1,2, \cdots, n$; $k=1,2, \cdots, n_{i}$, so that (i) $\Delta_{i}^{\prime \prime l} \subset \Delta_{i}$ if $l<n_{i}$, (ii) $\Delta_{i}^{\prime \prime n_{i}}$ contains the right-hand end point of $\Delta_{i}$ (a single $\Delta_{j}^{\prime \prime}$ is to be indexed in more than one way if it contains more than one such end point), (iii) $\Delta_{i}^{\prime \prime k_{1}}$ is to the left of $\Delta_{i}^{\prime \prime k_{2}}$ if $k_{1}<k_{2}$. Let

$$
\begin{aligned}
& \Delta_{i}^{\prime k}= \begin{cases}\Delta_{i-1}^{\prime \prime n_{i-1}} \cap \Delta_{i} & \text { if } k=0, \\
\Delta_{i}^{\prime \prime k} & \text { if } 1 \leqq k \leqq n_{i}-1, \\
\Delta_{i}^{\prime \prime n_{i}} \cap \Delta_{i} & \text { if } k=n_{i},\end{cases} \\
& t_{i}^{\prime k}=\left\{\begin{array}{lll}
t_{i}^{\prime \prime k} & \text { if } 1 \leqq k \leqq n_{i}-1, \\
\text { any element of } \Delta_{i}^{\prime \prime} & \text { if } \quad k=0 \text { or } n_{i} \text { and }
\end{array}\right. \\
& \text { any element of }[\alpha, \beta] \text { otherwise. }
\end{aligned}
$$

Those $\Delta_{i}^{\prime k}$ which are not empty determine an S-P, $\Pi^{\prime}$, which is a refinement of II. Moreover from Definition 4.1 we see that $\mu\{t, \sigma\}=e$ if $\sigma$ is empty so we have $z^{\prime}=\prod_{i=1}^{n} \prod_{k=0}^{n_{i}}, \mu\left\{t_{i}^{\prime k}, \Delta_{i}^{\prime k}\right\} \in J\left(\mu, \Pi^{\prime}\right)$. Let $z_{1}=\prod_{i=1}^{n} \prod_{k=1}^{n_{i}-1} \mu\left\{t_{i}^{\prime k}, \Delta_{i}^{\prime k}\right\}$.

Each factor in $z_{1}$ coincides with a factor in $z=\prod_{j=1}^{n^{\prime \prime}} \mu\left\{t_{j}^{\prime \prime}, \Delta_{j}^{\prime \prime}\right\}$ and only those factors for which $\Delta_{j}^{\prime \prime}$ contains the right-hand end point of some $\Delta_{i}$ are omitted. Inserting an $e$ in $z_{1}$ for each such factor and applying Definition 3.22 we have $\rho\left(z, z_{1}\right)<\epsilon$ provided 


$$
\begin{aligned}
& \sum_{j=1}^{n^{\prime \prime}} \rho\left(\mu\left\{t_{j}^{\prime \prime}, \Delta_{j}^{\prime \prime}\right\}, e\right)<B(\beta-\alpha), \\
& \sum \rho\left(\mu\left\{t_{j}^{\prime \prime}, \Delta_{i}^{\prime \prime}\right\}, e\right)<\delta_{B(\beta-\alpha)}(\epsilon)
\end{aligned}
$$

where the latter sum is taken over those $j$ for which $\Delta_{j}^{\prime \prime}$ contains a righthand end point of a $\Delta_{i}$.

If one assumes that $B$ is a bound for $\mu$, it follows from Definition 4.1 that (5.13) is always satisfied while $(5.14)$ is satisfied whenever $n \cdot\left|\Pi^{\prime \prime}\right|$ $<\delta_{B(\beta-\alpha)}(\epsilon)$. A similar argument shows that in this case $\rho\left(z^{\prime}, z_{1}\right)<\epsilon$ as well.

Hence, for any $\epsilon>0,\left|\Pi^{\prime \prime}\right|<\delta_{B(\beta-\alpha)}(\epsilon) / n$ implies that for any $z \in J\left(\mu, \Pi^{\prime \prime}\right), \rho(z, x) \leqq \rho\left(z, z_{1}\right)+\rho\left(z_{1}, z^{\prime}\right)+\rho\left(z^{\prime}, x\right)<3 \epsilon$. Thus (5.9) holds and the proof is complete.

Other assumptions might replace boundedness in the hypotheses of Theorem 5.8, but bounded variation is not enough, as the following example shows.

(5.15) ExAmple. Let $\mathfrak{D}$ be the additive group of real numbers, let $\mu\{t, \sigma\}=1$ if $t=1 / 2$ and $1 / 2 \in \sigma$, and let $\mu\{t, \sigma\}=0$ otherwise. Let $\Delta_{1}=[0,1 / 2), \Delta_{2}=[1 / 2,1 / 2], \Delta_{3}=(1 / 2,1]$. Then $J\left(\mu, \Pi^{\prime}\right)=S J\left(\mu, \Pi^{\prime}\right)=1$ for any $\Pi^{\prime}\left\langle\Pi: \Delta_{i}, i=1,2,3\right.$, while for any other S-P, $\Pi^{*}, J\left(\mu, \Pi^{*}\right)=S J\left(\mu, \Pi^{*}\right)$ $=\{0,1\}$.

(5.16) THEOREM. If $\mathfrak{D}$ is relatively stable and $\mu$ is a bounded differential on $[\alpha, \beta]$, then the following statements are equivalent:

$$
\begin{aligned}
(R) \int_{\alpha}^{\beta} \mu\{t, d t\} & =\lim _{|\Pi| \rightarrow 0} R J(\mu, \Pi)=x, \\
\lim _{\Pi \downarrow} R J(\mu, \Pi) & =x, \\
\lim _{R \downarrow} R J(\mu, R) & =x .
\end{aligned}
$$

Proof. The proof that (5.17) and (5.18) are equivalent is essentially similar to that of Theorem 5.8 and is omitted.

Since $R \prec \Pi(R)$, we have, from (5.3), $R J(\mu, R) \subset R J(\mu, \Pi(R))$. Thus (5.19) follows from (5.18). On the other hand any refinement of the S-P whose intervals are all intervals forming the sets $s_{i}^{j}$ of $R$ is a refinement of $R$ so (5.19) implies (5.18).

Note that (5.18) and (5.19) are equivalent and are implied by (5.17) whether $\mu$ :s bounded or not.

Finally from (5.1) we infer:

(5.20) ThEorem. If $(R) \int_{\alpha}^{\beta} \mu\{t, d t\}$ exists, then so do $(S) \int_{\alpha}^{\beta} \mu\{t, d t\}$ and $\int_{\alpha}^{\beta} \mu\{t, d t\}$, and the three integrals are equal.

6. Disjoint intervals and nonoverlapping intervals. In additive Riemann 
integration theory it is immaterial whether one considers partitions into disjoint intervals or partitions into intervals which are merely nonoverlapping. The example (5.15) shows that this is not the case for arbitrary abstract differentials.

(6.1) Convention. A symbol with an asterisk, *, after it is to have the same meaning as the symbol without the asterisk except that wherever partitions into disjoint intervals were used in defining the symbol, partitions into nonoverlapping intervals are to be used in defining the corresponding asterisked symbol.

For example, an $\mathrm{S}-\mathrm{P}^{*}$ of $[\alpha, \beta]$ is a representation of $[\alpha, \beta]$ as $\bigcup_{i=1}^{n} \Delta_{i}$ where the $\Delta_{i}$ are nonoverlapping intervals. We do not require the $\Delta_{i}$ to be closed so each $\mathrm{S}-\mathrm{P}$ is also an $\mathrm{S}-\mathrm{P}^{*}$.

(6.2) TheOREM. If $\mu$ is a differential on $[\alpha, \beta]$, a necessary condition that $\left(S^{*}\right) \int_{\alpha}^{\beta} \mu\{t, d t\}=x$ is that $(S) \int_{\alpha}^{\beta} \mu\{t, d t\}=x$. If

(6.3) $D$ is relatively stable,

(6.4) $\mu$ is approximately-multiplicative,

(6.5) $\mu$ is of bounded $R$-variation,

(6.6) $\mu\{t, \Delta\}=e$ whenever $\Delta$ consists of a single point,

(6.7) $\mu\{t, \Delta\}=\mu\left\{t, \Delta^{\prime}\right\}$ whenever $\Delta$ and $\Delta^{\prime}$ are intervals with the same end points,

then the condition is also sufficient.

Proof. The condition is necessary since $S J(\mu, \Pi) \subset S J^{*}(\mu, \Pi)$ for any S-P, II.

Let $\Pi^{*}$ be any $\mathrm{S}-\mathrm{P}^{*}$ and $y^{*}$ any element of $S J^{*}\left(\mu, \Pi^{*}\right)$. Then by Definition 3.17 and Convention 6.1

$$
y^{*}=\prod_{i=1}^{n^{\prime}} \mu\left\{t_{i}^{\prime}, \Delta_{i}^{\prime}\right\}
$$

where $\Pi^{\prime}: \Delta_{i}^{\prime}<\Pi^{*}$ and $t_{i}^{\prime} \in \Delta_{i}^{\prime}$. Consider all intervals of the form $U_{k=1}^{l} \Delta_{i+k}^{\prime}$ where $t_{i}^{\prime} \neq t_{i+1}^{\prime}=t_{i+2}^{\prime}=\cdots=t_{i+l}^{\prime} \neq t_{i+l+1}^{\prime}$. (If $l>2$, then $\Delta_{i+2}^{\prime}, \cdots, \Delta_{i+l-1}^{\prime}$ are all degenerate.) Take the interior of each such interval and unite it with the corresponding $t_{i+1}^{\prime}$. These intervals are disjoint and their closures cover $[\alpha, \beta]$. Accordingly we can form an $\mathrm{S}-\mathrm{P}, \Pi: \Delta_{j}$, by adding to each such interval its left-hand end point, unless this is an element of the next interval to the left. The point $\beta$ is to be added to the last interval. Clearly, $|\Pi|$ $\leqq 2\left|\Pi^{\prime}\right| \leqq 2\left|\Pi^{*}\right|$. If $\Delta_{i}^{\prime}$ is among those intervals used in forming $\Delta_{j}$, then $t_{i}^{\prime} \in \Delta_{j}$, and if several $\Delta_{i}^{\prime}$ are used in forming a single $\Delta_{j}$, then the corresponding $t_{i}^{\prime}$ are equal. Let $t_{j}=t_{i}^{\prime}$ if $\Delta_{i}^{\prime}$ is used in forming $\Delta_{j}$. Then

$$
y=\prod_{j=1}^{n} \mu\left\{t_{j}, \Delta_{i}\right\} \in S J(\mu, \Pi) .
$$


Each factor in (6.9) corresponds to a product $\prod_{k=1}^{l} \mu\left\{t_{i+k}^{\prime}, \Delta_{i+k}^{\prime}\right\}$ which appears as part of the product in (6.8). Moreover all the $t_{i+k}^{\prime}$ appearing in this product are equal to $t_{j}$ and not more than two of the $\Delta_{i+k}^{\prime}$ are nondegenerate. By (6.6), $\mu\left\{t_{i+k}^{\prime}, \Delta_{i+k}^{\prime}\right\}=e$ if $\Delta_{i+k}^{\prime}$ is degenerate so such terms may be neglected. By (6.7) the remaining factors in $\prod_{k=1}^{l} \mu\left\{t_{i+k}^{\prime}, \Delta_{i+k}^{\prime}\right\}$ are unchanged if we replace $\Delta_{i+k}^{\prime}$ by intervals with the same end points in such a way that the $\Delta_{i+k}^{\prime}$ are disjoint and their union is $\Delta_{j}$. Hence by Definition 4.8 the distance between the product of these factors in (6.8) and the corresponding factor $\mu\left\{t_{j}, \Delta_{j}\right\}$, in (6.9) is not more than $\eta\left(\left|\Delta_{i}\right|\right) \cdot\left|\Delta_{i}\right|$. Thus we group the factors in (6.8) so that there is a one-to-one correspondence between the groups and the factors in (6.9) and the sum of the distances between each group and its corresponding factor is not more than $(\beta-\alpha) \cdot \eta(|\Pi|)$ $\leqq(\beta-\alpha) \cdot \eta\left(2\left|\Pi^{*}\right|\right)$. Since $\mu$ is of bounded $R$-variation there is a $V$ such that $\sum_{i=1}^{n} \rho\left(\mu\left\{t_{i}, \Delta_{i}\right\}, e\right) \leqq V$. By Definition 3.22

$$
\rho\left(y^{*}, y\right)<\epsilon
$$

provided

$$
(\beta-\alpha) \cdot \eta\left(2\left|\Pi^{*}\right|\right)<\delta_{1}=\delta_{V}(\epsilon) .
$$

If $(S) \int_{\alpha}^{\beta} \mu\{t, d t\}=x$, then there is a $\delta_{2}$ such that $|\Pi|<\delta_{2}$ and $y \in S J(\mu, \Pi)$ imply $\rho(y, x)<\epsilon$. Choose $\delta$ so that $\delta<\delta_{2} / 2$, and $\left|\Pi^{*}\right|<\delta$ implies (6.11). Thus if $\left|\Pi^{*}\right|<\delta$, then $(6.10)$ obtains and $\rho(x, y)<\epsilon$ so $\rho\left(y^{*}, x\right)<2 \epsilon$, which completes the proof.

From Definitions 3.21 and 4.1 and 4.3 we infer:

(6.12) Theorem. Theorem 6.2 remains valid if the hypotheses (6.5), (6.6), and (6.7) are replaced by the hypothesis $\mu$ is bounded. It also remains valid if hypotheses (6.3) and (6.5) are replaced by the hypothesis $\mathfrak{D}$ is absolutely stable.

The situation in $R$-integration is more complicated and the most that we have been able to prove is the following.

(6.13) THEOREM. If $\mu$ is a differential on $[\alpha, \beta]$, a necessary condition that $\left(R^{*}\right) \int_{\alpha}^{\beta} \mu\{t, d t\}=x$ is that $(R) \int_{\alpha}^{\beta} \mu\{t, d t\}=x$. If $\mathfrak{D}$ is relatively stable and $\mu$ is bounded and approximately multiplicative, then the condition is sufficient.

The proof is similar to, but more cumbersome than that of Theorem 6.2.

7. Elementary properties.

(7.1) Theorem. If $\mu$ is a bounded differential on $[\alpha, \gamma], \alpha \leqq \beta \leqq \gamma$, and $\mathfrak{D}$ is relatively stable, then $(R) \int_{\alpha}^{\beta} \mu\{t, d t\}=x$, and $(R) \int_{\beta}^{\gamma} \mu\{t, d t\}=y$ imply (R) $\int_{\alpha}^{\gamma} \mu\{t, d t\}=x \cdot y$.

Proof. Consider any S-P, $\Pi$, of $[\alpha, \gamma]$ and any $z=\prod_{i=1}^{n} \prod_{j=1}^{n_{i}} \mu\left\{t_{i}^{j}, s_{i}^{j}\right\}$ $\in R J(\mu, \Pi)$. Determine $n^{\prime}, n^{\prime \prime}$ so that $\Delta_{i}=\bigcup_{j=1}^{n_{i}} s_{i}^{j} \subset[\alpha, \beta]$ if and only if $i \leqq n^{\prime}$, and $\Delta_{i} \subset[\beta, \gamma]$ if and only if $i \geqq n^{\prime \prime}$. Either $n^{\prime \prime}-n^{\prime}=1$ or $n^{\prime \prime}-n^{\prime}=2$. Let 
$\mu_{i}^{j}=\mu\left\{t_{i}^{j}, s_{i}^{j}\right\}, x_{1}=\prod_{i=1}^{n^{\prime}} \prod_{j=1}^{n_{i}} \mu_{i}^{j}, y_{1}=\prod_{i=n^{\prime \prime}}^{n} \prod_{j=1}^{n_{i}} \mu_{i}^{j}, z_{1}=\prod_{i=n^{\prime}+1}^{n^{\prime \prime}-1} \prod_{j=1}^{n_{i}} \mu_{i}^{j}$. Thus $z=x_{1} \cdot z_{1} \cdot y_{1}$.

It is possible to find $\Pi_{1}$ an S-P of $[\alpha, \beta]$ and $\Pi_{2}$ an S-P of $[\beta, \gamma]$ and sets $s^{\prime}$ and $s^{\prime \prime}$ and points $t^{\prime} \in s^{\prime}, t^{\prime \prime} \in s^{\prime \prime}$ so that $x_{2}=x_{1} \cdot \mu\left\{t^{\prime}, s^{\prime}\right\} \in R J\left(\mu, \Pi_{1}\right)$ and $y_{2}=\mu\left\{t^{\prime \prime}, s^{\prime \prime}\right\} \cdot y_{1} \in R J\left(\mu, \Pi_{2}\right)$ and $\left|\Pi_{1}\right| \leqq|\Pi|$ and $\left|\Pi_{2}\right| \leqq|\Pi|$.

By Definitions 3.20 and 3.22

$$
\rho\left(x \cdot y, x_{2} \cdot y_{2}\right)<\epsilon
$$

provided $\left|\Pi_{1}\right|$ and $\left|\Pi_{2}\right|$ are sufficiently small while

$$
\begin{aligned}
\rho\left(z, x_{2} \cdot y_{2}\right)=\rho\left(x_{1} \cdot e \cdot\left(\prod_{i=n^{\prime}+1}^{n^{\prime \prime}-1} \prod_{j=1}^{n_{i}} \mu_{i}^{j}\right) \cdot e \cdot y_{1},\right. \\
\left.x_{1} \cdot \mu\left\{t^{\prime}, s^{\prime}\right\} \cdot\left(\prod_{i=n^{\prime}+1}^{n^{\prime \prime}-1} \prod_{j=1}^{n_{i}} e\right) \cdot \mu\left\{t^{\prime \prime}, s^{\prime \prime}\right\} \cdot y_{1}\right)<\epsilon
\end{aligned}
$$

provided

$$
\sum_{i=1}^{n} \sum_{j=1}^{n_{i}} \rho\left(\mu_{i}^{j}, e\right) \leqq B(\gamma-\alpha)
$$

and

$$
\rho\left(\mu\left\{t^{\prime}, s^{\prime}\right\}, e\right)+\sum_{i=n^{\prime}+1}^{n^{\prime \prime}-1} \sum_{j=1}^{n_{i}} \rho\left(\mu_{i}^{j}, e\right)+\rho\left(\mu\left\{t^{\prime \prime}, s^{\prime \prime}\right\}, e\right) \leqq \delta_{B(\gamma-\alpha)}(\epsilon) .
$$

By Definition 4.1 equation (7.4) is automatically satisfied while (7.5) is satisfied if $3 B|\Pi|<\delta_{B(\gamma-\alpha)}(\epsilon)$. Thus (7.3) is satisfied for sufficiently small $|\Pi|$ and since $\left|\Pi_{1}\right|<|\Pi|$ and $\left|\Pi_{2}\right|<|\Pi|,(7.2)$ is also satisfied. Combining these, we see that for any $\epsilon>0$ we can ensure that $\rho(z, x \cdot y)<2 \epsilon$ for any $z \in R J(\mu, \Pi)$ by making $|\Pi|$ small enough.

Several variants of Theorem 7.1 are also true. The proofs are similar to that of Theorem 7.1 and are omitted.

(7.6) THEOREM. If $\mu$ is a bounded differential on $[\alpha, \gamma], \alpha \leqq \beta \leqq \gamma$, and $\mathfrak{D}$ is relatively stable, then $(S) \int_{\alpha}^{\beta} \mu\{t, d t\}=x,(S) \int_{\beta}^{\gamma} \mu\{t, d t\}=y$ imply $(S) \int_{\alpha}^{\gamma} \mu\{t, d t\}$ $=x \cdot y$.

(7.7) TheOREM. In Theorem 7.6 the hypothesis that $\mu$ is bounded may be replaced by $\mu$ is of bounded variation and $\lim _{\Delta \rightarrow \beta} \mu\{t, \Delta\}=e$.

(7.8) TheOREM. In Theorem 7.7 the hypothesis $\mu$ is of bounded variation may be dropped if $\mathfrak{D}$ is absolutely stable.

On the other hand, the existence of an integral over $[\alpha, \beta]$ need not imply its existence over a smaller interval.

(7.9) EXAMPLE. Let $\mathfrak{D}$ be the real numbers with the operation of multipli- 
cation. Let $f(t)=1$ if $t$ is rational and 0 otherwise. Let

$$
\mu\{t, \sigma\}=\left\{\begin{array}{l}
0 \text { if } t \in \sigma \text { and } 1 \in \sigma, \\
1+f(t)|\sigma| \text { otherwise. }
\end{array}\right.
$$

This differential is approximately multiplicative and of bounded variation on any finite interval, but $(S) \int_{\alpha}^{\beta} \mu\{t, d t\}$ exists if and only if $1 \in[\alpha, \beta]$.

(7.10) THEOREM. If $\mu$ is a bounded differential on $[\alpha, \beta]$, and $\mathfrak{D}$ is relatively stable, and $(S) \int_{\alpha}^{\gamma} \mu\{t, d t\}$ exists for all $\gamma, \alpha \leqq \gamma \leqq \beta$, then

$$
\lim _{\gamma \rightarrow \alpha+}(S) \int_{\alpha}^{\gamma} \mu\{t, d t\}=e .
$$

Proof. Let $B$ be a bound for $\mu$ and let $\epsilon>0$ be given. For any $\gamma, \alpha \leqq \gamma \leqq \beta$, there is an S-P, $\Pi: \Delta_{i}$, of $[\alpha, \gamma]$ and a choice of $t_{i} \in \Delta_{i}$ so that

$$
\rho\left((S) \int_{\alpha}^{\gamma} \mu\{t, d t\}, \prod_{i=1}^{n} \mu\left\{t_{i}, \Delta_{i}\right\}\right)<\epsilon .
$$

By Definition 3.22, if $\gamma-\alpha<\delta_{B(\beta-\alpha)}(\epsilon) / B$, then $\rho\left(\prod_{i=1}^{n} \mu\left\{t_{i}, \Delta_{i}\right\}, e\right)<\epsilon$. Thus, for $\gamma$ sufficiently close to $\alpha, \rho\left(e,(S) \int_{\alpha}^{\gamma} \mu\{t, d t\}\right)<2 \epsilon$.

By similar methods we can obtain:

(7.11) TheOREM. If $\mu$ is a bounded differential on $[\alpha, \beta]$ and $\mathfrak{D}$ is relatively stable and $(S) \int_{\alpha}^{\gamma} \mu\{t, d t\}$ exists for all $\gamma$ in a neighborhood of $\gamma_{0}\left(\alpha<\gamma_{0}<\beta\right)$, then $(S) \int_{\alpha}^{\gamma} \mu\{t, d t\}$ is a continuous function of $\gamma$ at $\gamma_{0}$.

(7.12) Theorem. Both Theorem 7.10 and Theorem 7.11 remain valid if $S$-integration is replaced by $R$-integration.

8. Existence of $R$-integrals. From Theorem 5.7 we know that a sufficient condition that $(R) \int_{\alpha}^{\beta} \mu\{t, d t\}$ exist is that $d(R J(\mu, \Pi))$ can be made arbitrarily small by making $|\Pi|$ small enough. We proceed to calculate an upper bound for $R J(\mu, \Pi)$.

Let $\Pi: \Delta_{i}, i=1,2, \cdots, n$, be given and choose $t_{i} \in \Delta_{i}$. Let $\mu_{i}=\mu\left\{t_{i}, \Delta_{i}\right\}$ and let $x=\prod_{i=1}^{n} \mu_{i}$. Let $x^{\prime}=\prod_{i=1}^{n \prime} \prod_{j=1}^{n_{i}} \mu\left\{t_{i}^{\prime j}, s_{i}^{\prime j}\right\}$ be any element of, $R J(\mu, \Pi)$. There is no loss of generality in assuming that $n^{\prime}=n$ and $\bigcup_{j=1}^{n_{i}} s_{i}^{\prime j}=\Delta_{i}$. Let $\mu_{i}^{\prime j}=\mu\left\{t_{i}^{\prime j}, s_{i}^{\prime j}\right\}, \mu_{i}^{* j}=\mu\left\{t_{i}, s_{i}^{\prime j}\right\}$, and let $x^{*}=\prod_{i=1}^{n} \prod_{j=1}^{n_{i}} \mu_{i}^{* j}$.

If $\mathfrak{D}$ is relatively stable and $\mu$ is of bounded $R$-variation, then, by Definitions 3.22 and 4.2 , for any $\epsilon>0$ there is a $\delta_{V}(\epsilon)$ such that

$$
\rho\left(x, x^{*}\right)<\epsilon \text { and } \rho\left(x^{*}, x^{\prime}\right)<\epsilon
$$

provided

$$
\sum_{i=1}^{n} \rho\left(\mu_{i}, \prod_{j=1}^{n_{i}} \mu_{\mu_{i}^{* j}}^{* j}\right)<\delta_{V}(\epsilon)
$$


and

$$
\sum_{i=1}^{n} \sum_{j=1}^{n_{i}} \rho\left(\mu_{i}^{* j}, \mu_{i}^{\prime j}\right)<\delta_{V}(\epsilon)
$$

respectively.

If $\mu$ is approximately multiplicative, it follows from Definition 4.8 that (8.2) is satisfied provided

$$
\eta(|\Pi|)<\delta_{V}(\epsilon) /(\beta-\alpha) .
$$

From Definition 4.4 we see that (8.3) is satisfied whenever for some nonnegative additive set function, $m$,

$$
\sum_{i=1}^{n} \operatorname{Osc}_{m}\left(\mu \mid \Delta_{i}\right) m\left(\Delta_{i}\right)<\delta_{V}(\epsilon)
$$

Let $F_{m, \Pi}(t)=\operatorname{Osc}_{m}\left(\mu \mid \Delta_{i}\right)$ whenever $t \in \Delta_{i}$. Let

$$
F_{m, \zeta}(t)=\sup _{|\mathbf{I}|<\zeta} F_{m, \mathrm{II}}(t) .
$$

Since $F_{n, \mathrm{II}}$ is a step function and the domain of $m$ includes all intervals, $F_{m, \mathrm{II}}$ is measurable with respect to $m$ and (8.5) may be rewritten as $\int_{\alpha}^{\beta} F_{m, \Pi}(t) d m(t)$ $<\delta_{V}(\epsilon)$ which is clearly implied by

$$
\int_{\alpha}^{\beta} F_{m, \zeta}(t) d m(t)<\delta_{V}(\epsilon)
$$

provided $|\Pi|<\zeta$ and $F_{m, \zeta}(t)$ is summable with respect to $m$. It is easily seen from Definition 4.4 that $\left.\sup _{\mid \mathrm{II}}\right|_{<\zeta} F_{m, \mathrm{II}}(t)$ is unchanged if, in forming the supremum, we consider only S-P's whose intervals have rational end points (except possibly $\alpha$ and $\beta$ ). As this class is enumerable it follows that $F_{m, \zeta}(t)$ is measurable. From Definition 4.5 we infer that $\lim _{\zeta \rightarrow 0} F_{m, 5}(t)=\operatorname{Osc}_{m}(\mu \mid t)$ while from (8.6) it is clear that $F_{m, \zeta}(t)$ is a nonincreasing function of $\zeta$.

Hence the conditions Osc $(\mu \mid t)=0$ for all $t \in[\alpha, \beta]-E$ where $m(E)=0$ and $\int_{\alpha}^{\beta} F_{m, \zeta_{0}}(t) d m(t)<+\infty$ for some $\zeta_{0}>0$ imply the existence of a $\delta^{\prime}(\epsilon)$ such that $\zeta<\delta^{\prime}(\epsilon)$ implies (8.7) and hence (8.5) and (8.3). Combining this with the fact that (8.4) implies (8.2) we see that (8.1) holds for all sufficiently small $|\Pi|$. As $x^{\prime}$ was an arbitrary element of $R J(\mu, \Pi)$ and $x$ was chosen independently of $x^{\prime}$ it follows that for all sufficiently small $|\Pi|, d(R J(\mu, \Pi)<4 \epsilon$. This completes the proof of the following theorem.

(8.8) THEOREM. If $\mathfrak{D}$ is relatively stable, and if $\mu$ is a differential approximately multiplicative and of bounded $R$-variation on $[\alpha, \beta]$, and if, for some non-negative additive set function $m, \operatorname{Osc}_{m}(\mu \mid t)=0$ for all $t \in[\alpha, \beta]-E$ where $m(E)=0$, and if $\int_{\alpha}^{\beta} F_{m, \zeta_{0}}(t) d m(t)<+\infty$ for some $\zeta_{0}>0$, then $(R) \int_{\alpha}^{\beta} \mu\{t, d t\}$ exists. 
We shall use Theorem 8.8 in $\$ 11$, but for bounded differentials we have a simpler result.

(8.9) THEOREM. If $\mathfrak{D}$ is relatively stable, and $\mu$ is a bounded, approximately multiplicative differential on $[\alpha, \beta]$, and $\operatorname{Osc}(\mu \mid t)=0$ for almost all $t \in[\alpha, \beta]$, then $(R) \int_{\alpha}^{\beta} \mu\{t, d t\}$ exists.

Proof. From Definition 4.1 it follows that if $m$ is Lebesgue measure, then $F_{m, 5}(t) \leqq 2 B$ where $B$ is a bound for $\mu$. Using this and (4.3) we see that the hypotheses of Theorem 8.8 are satisfied.

In the proof of Theorem 8.8 the assumption that $\mu$ is of bounded $R$ variation is needed only in inferring (8.1) from (8.2) and (8.3). Accordingly we also have the following.

(8.10) ThEOREM. If $\mathfrak{D}$ is absolutely stable, the hypothesis that $\mu$ is of bounded $R$-variation may be omitted in Theorem 8.8.

By virtue of Theorem 5.20 we have:

(8.11) Theorem. Theorems $8.8,8.9$, and 8.10 remain valid if $(R) \int_{\alpha}^{\beta} \mu\{t, d t\}$ is replaced by $(S) \int_{\alpha}^{\beta} \mu\{t, d t\}$ or by $\int_{\alpha}^{\beta} \mu\{t, d t\}$.

\section{Part III. Lebesgue InTEgration}

9. Elementary properties. We now state explicitly the meaning of the infinite products used in the definition (3.19) of Lebesgue integral range.

(9.1) Definition. If $B_{i}, i=1,2, \cdots, n$, are linearly ordered sets and $x_{i}^{b} \in \mathfrak{D}$ for each $b \in B_{i}$, then $\prod_{i=1}^{n} \prod_{b \in B_{i}} x_{i}^{b}=x$ means given any neighborhood, $\mho$, of $x$ there are finite sets $C_{i} \subset B_{i}$ such that if $D_{i}$ are finite sets and $C_{i} \subset D_{i}$ $\subset B_{i}$, then $\prod_{i=1}^{n} \prod_{b \in D_{i}} x_{i}^{b} \in \mathcal{U}$ where the order of the factors in $\prod_{b \in D_{i}} x_{i}^{b}$ coincides with the order of their superscripts.

From Definition 3.19, (1.1), (1.2), and (2.7) we obtain:

(9.2) If $L J(\mu, L)$ exists and $L^{\prime}<L$, then $L J\left(\mu, L^{\prime}\right)$ exists and $L J\left(\mu, L^{\prime}\right)$ $\subset L J(\mu, L)$.

(9.3) If $L J(\mu, L)$ exists and $L_{1}$ and $L_{2}$ are refinements of $L$, then $L J\left(\mu, L_{1}\right)$ $\cap L J\left(\mu, L_{2}\right)$ is not void.

(9.4) Theorem. If $L J(\mu, L)$ exists for some $\mathrm{L}-\mathrm{P}, L$, of $[\alpha, \beta]$, then a necessary and sufficient condition that $(L) \int_{\alpha}^{\beta} \mu\{t, d t\}$ exist is that $\inf _{L^{\prime}}<L d\left(L J\left(\mu, L^{\prime}\right)\right.$ $=0$.

(9.5) Theorem. If $L J(\mu, L)$ exists for some L-P, $L$, of $[\alpha, \beta]$ and $(L) \int_{\alpha}^{\beta} \mu\{t, d t\}$ exists, then $(L) \int_{\alpha}^{\beta} \mu\{t, d t\}=\bigcap_{L^{\prime}} \prec L \mathrm{Cl}\left(L J\left(\mu, L^{\prime}\right)\right)$ where $\mathrm{Cl}\left(L J\left(\mu, L^{\prime}\right)\right)$ is the closure of $L J\left(\mu, L^{\prime}\right)$.

(9.6) Definition. A differential $\mu$ on $[\alpha, \beta]$ is of bounded $L$-variation if there is a number $V$ such that $\sum_{i=1}^{n} \sum_{b \in B_{i}} \rho\left(\mu\left\{t_{i}^{b}, \sigma_{i}^{b}\right\}, e\right) \leqq V$ for all L-P's, $L: \sigma_{i}^{b}$, of $[\alpha, \beta]$ and all $t_{i}^{b} \in \sigma_{i}^{b}$. 
(9.7) If a differential on $[\alpha, \beta]$ is bounded, then it is of bounded $L$-variation.

(9.8) THEOREM. If $\mathfrak{D}$ is relatively stable and $\mu$ is a differential of bounded $L$-variation on $[\alpha, \beta]$, then $L J(\mu, L)$ exists for any L-P, $L$, of $[\alpha, \beta]$.

Proof. We must show that for any L-P, $L: \sigma_{i}^{b}$, and any $t_{i}^{b} \in \sigma_{i}^{b}$ the product $\prod_{i=1}^{n} \prod_{b \in B_{i}} \mu\left\{t_{i}^{b}, \sigma_{i}^{b}\right\}$ exists. Since relative stability implies continuity of multiplication, it is enough to show that, for each $i, \prod_{b \in B_{i}} \mu^{b}$ exists where $\mu^{b}=\mu\left\{t_{i}^{b}, \sigma_{i}^{b}\right\}$. By Definition 9.6, $\sum_{b \in B_{i}} \rho\left(\mu^{b}, e\right) \leqq V$. Let $\delta_{V}\left(1 / 2^{n}\right)$ be chosen as in Definition 3.22. Since $\sum_{b \in B_{i}} \rho\left(\mu^{b}, e\right)$ is convergent, we can choose finite sets $C_{\nu} \subset B_{i}, \nu=1,2, \cdots$, such that $C_{\nu} \subset C_{\nu+1}$ and, for any finite set $C$ containing $C_{\nu}, \sum_{b \in C-C_{\nu}} \rho\left(\mu^{b}, e\right)<\delta_{V}\left(1 / 2^{\nu}\right)$. Let $x_{\nu}=\prod_{b \in C} \mu^{b}$. By Definition $3.22, \rho\left(x_{\nu}, x_{\nu+k}\right)<1 / 2^{\nu}$. Thus $x_{1}, x_{2}, \cdots$ is a Cauchy sequence and has a limit $x$; moreover $\rho\left(x, x_{\nu}\right) \leqq 1 / 2^{\nu}$. Now given $\epsilon>0$, choose $\nu$ so that $\left(1 / 2^{\nu}\right)<\epsilon / 2$. If $C$ is any finite set containing $C_{\nu}$, it follows from Definition 3.22 that $\rho\left(\prod_{b \in C} \mu^{b}, x\right) \leqq \rho\left(\prod_{b \in C} \mu^{b}, x_{v}\right)+\rho\left(x, x_{v}\right)<\epsilon$.

(9.9) Theorem. If $\mu$ is a differential on $[\alpha, \gamma], \alpha \leqq \beta \leqq \gamma, \mu\{\beta,\{\beta\}\}$ $\cdot \mu\{\beta, \quad\{\beta\}\}=\mu\{\beta, \quad\{\beta\}\}$ and multiplication in $\mathfrak{D}$ is continuous, then $(L) \int_{\alpha}^{\beta} \mu\{t, d t\}=x$ and $(L) \int_{\beta}^{\gamma} \mu\{t, d t\}=y$ imply $(L) \int_{\alpha}^{\gamma} \mu\{t, d t\}=x \cdot y$.

The proof of Theorem 9.9 is like that of Theorem 7.1 but the hypotheses are weaker since we need only consider L-P's, $L$, such that the degenerate interval $\{\beta\}$ is one of the intervals of $\Pi(L)$.

10. Relation between Riemann and Lebesgue integration. The proof [Birkhoff 1, p. 377] that, in a Banach space, additive Lebesgue integration includes additive Riemann integration is not applicable to our differentials, and the following theorem is proved by a process of approximating measurable sets by finite unions of intervals.

(10.1) THEOREM. If $\mathfrak{D}$ is relatively stable and $\mu$ is a bounded, approximately multiplicative differential on $[\alpha, \beta]$ and $(R) \int_{\alpha}^{\beta} \mu\{t, d t\}=x$, then

$$
\text { (L) } \int_{\alpha}^{\beta} \mu\{t, d t\}=x .
$$

We first show that the following lemma implies the theorem.

(10.2) Lemma. Given any $\delta>0$ and any L-P, $L: \sigma_{i}^{a}, i=1,2, \cdots, n ; a \in A_{i}$ with $\Pi(L)=\Pi: \Delta_{i}$ and any $t_{i}^{a} \in \sigma_{i}^{a}$, then there are finite sets $B_{i}=\left\{a_{i}^{1}, a_{i}^{2}, \cdots, a_{i}^{n_{i}}\right\}$ $C A_{i}$ and an R-P, $R: s_{i}^{j}, i=1,2, \cdots, n ; j=0,1, \cdots, n_{i}$ such that

$$
\begin{array}{r}
\Pi(R)=\Pi, \\
\sum_{i=1}^{n} \sum_{a \in A_{i}-B_{i}}\left|\sigma_{i}^{a}\right|<\delta,
\end{array}
$$




$$
\begin{gathered}
\sum_{i=1}^{n}\left|s_{i}^{0}\right|<\delta, \\
\sum_{i=1}^{n} \sum_{j=1}^{n_{i}}\left|s_{i}^{j}-\left(s_{i}^{j} \cap \sigma_{i}^{j}\right)\right|<\delta, \\
\sum_{i=1}^{n} \sum_{j=1}^{n_{i}}\left|\sigma_{i}^{j}-\left(s_{i}^{j} \cap \sigma_{i}^{j}\right)\right|<\delta, \\
t_{i}^{j} \in s_{i}^{j},
\end{gathered}
$$

where $\sigma_{i}^{j}=\sigma_{i}^{a_{i}^{j}}$ and $t_{i}^{j}=t_{i}^{a_{i}^{j}}$.

Proof of the theorem. Let $\epsilon>0$ be given. Let $B$ be a bound for $\mu$, let $\delta_{B(\beta-\alpha)}(\epsilon)$ be the $\delta$ of Definition 3.22, and let $\eta$ be the null function of Definition 4.8.

Since $(R) \int_{\alpha}^{\beta} \mu\{t, d t\}=x$, there is an S-P $\Pi: \Delta_{i}, i=1,2, \cdots, n$, such that both

$$
(\beta-\alpha) \eta(|\Pi|)<\delta_{B(\beta-\alpha)}(\epsilon)
$$

and $z \in R J(\mu, \Pi)$ implies $\rho(z, x)<\epsilon$. If $y$ is any element of $L J(\mu, \Pi)$, then

$$
y=\prod_{i=1}^{n^{\prime}} \prod_{a \in A_{i}} \mu\left\{\begin{array}{c}
a \\
t_{i}, \sigma_{i}
\end{array}\right\}
$$

where $L: \sigma_{i}^{a}<\Pi$. Moreover, there is no loss of generality in assuming that $n^{\prime}=n$ and $\Pi(L)=\Pi$. Let the sets $B_{i}$ and the R-P, $R: s_{i}^{\prime}$, be chosen according to Lemma 10.2 with $\delta=\delta_{B(\beta-\alpha)}(\epsilon) / B$.

Let

$$
\begin{aligned}
y_{1} & =\prod_{i=1}^{n} \prod_{j=1}^{n_{i}} \mu\left\{\begin{array}{c}
j \\
t_{i}, \sigma_{i}^{j}
\end{array}\right\} \\
z_{1} & =\prod_{i=1}^{n} \prod_{j=1}^{n_{i}} \mu\left\{t_{i}^{j}, s_{i}^{j}\right\}, \\
y_{2} & =\prod_{i=1}^{n} \prod_{j=1}^{n_{i}}\left[\mu\left\{t_{i}^{j}, \sigma_{i}^{j}-\left(s_{i}^{j} \cap \sigma_{i}^{j}\right)\right\} \cdot \mu\left\{t_{i}^{j}, s_{i}^{j} \cap \sigma_{i}^{j}\right\}\right], \\
z_{2} & =\prod_{i=1}^{n} \prod_{j=1}^{n_{i}}\left[\mu\left\{t_{i}^{j}, s_{i}^{j}-\left(s_{i}^{j} \cap \sigma_{i}^{j}\right)\right\} \cdot \mu\left\{t_{i}^{j}, s_{i}^{j} \cap \sigma_{i}^{j}\right\}\right], \\
y^{*} & =\prod_{i=1}^{n} \prod_{j=1}^{n_{i}} \mu\left\{t_{i}^{j}, s_{i}^{j} \cap \sigma_{i}^{j}\right\}, \\
z & =\prod_{i=1}^{n} \prod_{j=0}^{n_{i}} \mu\left\{t_{i}^{j}, s_{i}^{j}\right\},
\end{aligned}
$$


where $t_{i}^{0}$ is any element of $s_{i}^{0}$. From (10.3) and (10.8), $z \in R J(\mu, \Pi)$ so $\rho(x, z)<\epsilon$.

From (10.5) and Definition (4.1)

$$
\sum_{i=1}^{n} \rho\left(\mu\left\{t_{i}^{0}, s_{i}^{0}\right\}, e\right) \leqq B \sum_{i=1}^{n}\left|s_{i}^{0}\right|<\delta_{B(\beta-\alpha)}(\epsilon)
$$

whence, by Definition $3.22, \rho\left(z, z_{1}\right)<\epsilon$.

Similarly using in turn (10.6), (10.7), and (10.4) instead of (10.5) we obtain $\rho\left(z_{2}, y^{*}\right)<\epsilon, \rho\left(y^{*}, y_{2}\right)<\epsilon$, and $\rho\left(y_{1}, y\right)<\epsilon$.

Also from (10.9) and Definition 4.8

$$
\begin{aligned}
\sum_{i=1}^{n} \sum_{j=1}^{n_{i}} \rho\left(\mu\left\{t_{i}^{j}, s_{i}^{j}\right\}, \mu\left\{t_{i}^{j}, s_{i}^{j}-\left(s_{i}^{j} \cap \sigma_{i}^{j}\right)\right\} \mu\left\{t_{i}^{j}, s_{i}^{j} \cap \sigma_{i}^{j}\right\}\right) \\
\leqq \sum_{i=1}^{n} \sum_{j=1}^{n_{i}} \eta\left(\left|s_{i}^{j}\right|\right) \cdot\left|s_{i}^{j}\right| \\
\leqq \eta(|\Pi|)(\beta-\alpha)<\delta_{B(\beta-\alpha)}(\epsilon) .
\end{aligned}
$$

Whence, by Definition 3.22, $\rho\left(z_{1} z_{2}\right)<\epsilon$. Similarly $\rho\left(y_{1}, y_{2}\right)<\epsilon$, whence $\rho(y, x)$ $<7 \epsilon$.

Since $y$ was an arbitrary member of $L J(\mu, \Pi)$, this completes the proof of the theorem.

We omit the proof of Lemma 10.2 which consists of approximating the $\sigma_{i}^{a}$ from within by closed sets and then approximating these by finite unions of intervals.

\section{PART IV. Applications}

11. Additive integration. In this section we shall discuss the relation of our abstract system to additive integrals of familiar types. No new results are obtained, but a few well known theorems are shown to be special cases of our theorems (8.9) and (8.10).

The simplest realization of our abstract system is that already considered where $\mathfrak{D}$ is the linear space of real numbers and $\mu\{t, \sigma\}=f(t) \cdot|\sigma|$ where $f$ is a real-valued function. For our present purposes, however, this case is no easier to treat than that where $\mathfrak{D}$ is an arbitrary Banach space with norm \|\| and $f$ has its range in $\mathfrak{D}$. If $\mu$ has the above form we shall say that it is additive differential, it being understood that $\mathfrak{D}$ is a Banach space. The following are immediately verified by reference to (3.23), (4.1), (4.5), (4.8).

(11.1) If $\mu$ is an additive differential, $\mathfrak{D}$ is absolutely stable.

(11.2) If $\mu\{t, \sigma\}=f(t) \cdot|\sigma|$ is an additive differential, $\mu$ is bounded if and only if $f$ is bounded.

(11.3) If $\mu\{t, \sigma\}=f(t) \cdot|\sigma|$ is an additive differential, then Osc $(\mu \mid t)=0$ if and only if $f$ is continuous at $t$.

(11.4) If $\mu$ is an additive differential, then it is approximately multiplicative. 
We also observe:

(11.5) If $\mu$ is an additive differential and $\int_{\alpha}^{\beta} \mu\{t, d t\}$ exists, then so does (R) $\int_{\alpha}^{\beta} \mu\{t, d t\}$.

Proof. The proof follows from three theorems given by Birkhoff [1, Theorems 3, 4, and 9, p. 364]. From the first two we infer easily that the closed convex hull of $J(\mu, \Pi)$ tends to $\int_{\alpha}^{\beta} \mu\{t, d t\}$ as $|\Pi|$ tends to zero, and from the latter we see that $R J(\mu, \Pi)$ is included in the closed convex hull of $J(\mu, \Pi)$.

An inspection of Definitions 2.3, 3.16, and 3.20 shows that if $\mu\{t, \sigma\}$ $=f(t) \cdot|\sigma|$ is an additive differential, then $\int_{\alpha}^{\beta} \mu\{t, d t\}$ is just the integral $\int_{\alpha}^{\beta} f(t) d t$ defined by Graves [1, p. 166]. In view of (11.5) and (5.20) this yields the following theorem.

(11.6) Theorem. If $\mu$ is the additive differential $\mu\{t, \sigma\}=f(t) \cdot|\sigma|$, then Graves' integral $\int_{\alpha}^{\beta} f(t) d t$ and the integrals $\int_{\alpha}^{\beta} \mu\{t, d t\},(S) \int_{\alpha}^{\beta} \mu\{t, d t\}$ and $(R) \int_{\alpha}^{\beta} \mu\{t, d t\}$ coincide.

Moreover by Theorems 11.6 and 8.9 and (11.1), (11.2), (11.3), and (11.4) we obtain Graves' Theorem 2 [op. cit. p. 168], namely:

(11.7) If $f(t)$ is bounded and continuous almost everywhere on $[\alpha, \beta]$, then Graves' integral $\int_{\alpha}^{\beta} f(t) d t$ exists.

Since addition in a Banach space is commutative, we need not pay attention to the ordering of terms in finite sums. For infinite sums our Definition 9.1 yields the unconditional sum. Accordingly our restriction to integrals over an interval is no longer essential.

Our definition of a Lebesgue integral (denoted by $(L) \int \varsigma \mu\{t, d t\}$ ) is meaningful when $L: \sigma_{i}^{a}$ is interpreted as a representation of a space $\mathfrak{S}$ as a union of an enumerable number of disjoint sets, $\sigma_{i}^{a}$, belonging to a fixed $\sigma$-field $\mathfrak{M}$ of measurable subsets of $\mathfrak{S}$. We then have:

(11.8) Theorem. If $\mu$ is the additive differential, $\mu\{t, \sigma\}=f(t) \cdot|\sigma|$ if $\sigma \subset \mathfrak{M}, t \in \Im$, then $(L) \int \Im \mu\{t, d t\}$ is the integral $J(f)$ defined by Birkhoff $[1$, Definition 4, p. 367].

Proof. A reference preceded by a [B] is to Birkhoff's paper [1].

Assume that $(L) \int_{\varsigma \mu}\{t, d t\}$ exists. Then, by Theorem 9.4 for some L-P, $L: \sigma_{i}^{a}, d(L J(\mu, L))<1 / n$. Let $x_{0}$ be any element of $L J(\mu, L)$. From Definition 3.19, $\left\|\sum_{i=1}^{n} \sum_{a \in A_{i}} f\left(t_{i}^{a}\right) \cdot\left|\sigma_{i}^{a}\right|\right\| \leqq\left\|\sum_{i=1}^{n} \sum_{a \in A_{i}} f\left(t_{i}^{a}\right)\left|\sigma_{i}^{a}\right|-x_{0}\right\|+\left\|x_{0}\right\|<1 / n$ $+\left\|x_{0}\right\|$. This clearly implies that $f$ is summable under $L$ in the sense of [B] Definition 1. From Definition 3.19 and [B] Definition 2 it follows that $J_{L}(f)$ is contained in the closed convex hull of $L J(\mu, L)$ so $d\left(J_{L}(f)\right)<1 / n$ and $f$ is integrable in the sense of [B] Definition 3. That $(L) \int \varsigma \mu\{t, d t\}$ and $J(f)$ are equal is an immediate consequence of Theorems $9.4,9.5,[B] 12$, and [B] Definition 4. 
Conversely assume $J(f)$ exists. Then, for some L-P, $L$, of $\mathfrak{S}, d\left(J_{L}(f)\right)<1 / n$. From Definition 3.19 and [B] Theorem 9 and [B] Definition 2 we infer that $L J(\mu, L) \subset J_{L}(f)$ and from Theorem 9.4 we infer that $(L) \int \varsigma \mu\{t, d t\}$ exists.

Every function which is integrable in the sense of Graves obviously must be bounded. Thus from (11.1), (11.2), (11.4) and Theorems 10.1, 11.6, and 11.8 we obtain the following result given by Birkhoff $[1$, p. 377].

(11.9) If a function is integrable in the sense of Graves, it is integrable in the sense of Birkhoff.

We can also obtain an integral containing that defined by Price [1, p. 22]. References to that paper will be preceded by $[\mathrm{P}]$, but the reader will be assumed to be familiar with the symbols $C^{*}([\mathrm{P}]$ p. 20), $\tau(\sigma)([\mathrm{P}](8.1))$ and $([\mathrm{P}](8.2))$, and $(\mathfrak{X}) \int_{\varsigma} F(t) d \tau(t)([\mathrm{P}](13.8))$.

Let $\mathfrak{D}$ consist of the set of all closed, bounded subsets of a Banach space $B$. Let the $x \cdot y, x, y \in \mathfrak{D}$, be the closure of the set of all sums $\xi+\eta$ with $\xi \in x$, $\eta \in y$. It is easy to prove that this operation is associative and that $\prod_{i=1}^{n} x_{i}$ is the closure of the set of all sums of the form $\sum_{i=1}^{n} \xi_{i}$ with $\xi_{i} \in x_{i}$. Let $\rho(x, y)$ be the Hausdorff distance between $x$ and $y$ [Hausdorff 1, p. 146].

(11.10) Theorem. If $\mu\{t, \sigma\}=\mu\{\sigma\}$ is the closure of $\tau(\sigma) C^{*}(F(\sigma))$ and $F$ is integrable in the sense of Price, then $(L) \int \varsigma \mu\{t, d t\}$ exists and is the set whose sole member is $(\mathfrak{X}) \int \Subset F(t) d \tau(t)$.

Proof. Comparing Definitions 9.1, [P] (4.7), and [P] (4.8), we see that a product $\prod_{i=1}^{\infty} x_{i}, x_{i} \in \mathfrak{D}$, exists if and only if $\sum_{i=1}^{\infty} x_{i}$ converges weakly unconditionally to a bounded set.

From $[\mathrm{P}](10.2)$ and $[\mathrm{P}](11.1)$ and Theorem $[\mathrm{P}](10.3)$ and $[\mathrm{P}](10.4)$ it follows that there is an L-P $L: \sigma_{i}$ such that for any L-P: $L^{\prime}: \sigma_{j}^{\prime} \prec L$, $\sum_{i=1}^{\infty} \tau\left(\sigma_{j}^{\prime}\right) C^{*}\left(F\left(\sigma_{j}^{\prime}\right)\right)$ is strongly unconditionally convergent. But if a series of sets is strongly unconditionally convergent, so is the series of the closures of the sets. By Theorem [P] (5.12) the product $\prod_{i=1}^{\infty} \mu\left\{\sigma_{j}^{\prime}, t_{j}^{\prime}\right\}$ exists and is equal to the closure of the integral range $I\left(F, \mathfrak{S}, L^{\prime}\right)$ provided the latter is bounded. From Definition $[\mathrm{P}](11.1)$ and Theorem $[\mathrm{P}](10.4)$ there is an $L$ such that, for all $L^{\prime}<L, I\left(F, \widetilde{S}, L^{\prime}\right)$ is bounded, so from Theorem [P] (10.4) and Definition 3.19 for all such $L^{\prime}, L J\left(\mu, L^{\prime}\right)$ is the closure of $I\left(F, \widetilde{S}, L^{\prime}\right)$. It follows at once from Theorems $[\mathrm{P}](10.4)$ and $[\mathrm{P}](11.2)$ and Definitions 3.20 and $[\mathrm{P}](11.1)$ that $(L) \int \varsigma \mu\{d, d t\}$ is the set consisting of the single point $(\mathfrak{X}) \int \Im F(t) d \tau(t)$.

The integrals of functions with values in a general convex linear space studied by Phillips [1] and Rickart [1] can be obtained by a much more drastic modification of our system in two directions.

First we replace the assumption that $\mathfrak{D}$ has a metric topology by the assumption that the topology is given by a family of "pseudo metrics." That is, we assume the existence of a family, $A$, of real-valued functions, $\rho_{\alpha}$, with domain $D \times D$ such that $\rho_{\alpha}(x, y)$ satisfies all the usual axioms for a 
metric except that the axiom " $\rho(x, y)=0$ implies $x=y$ " is replaced by "the totality of sets of the form: the set of all $y$ such that $\rho_{\alpha}(x, y)<\epsilon, \rho_{\alpha} \in \mathcal{C}$, $x \in \mathfrak{D}, \epsilon>0$, form an open basis in $\mathfrak{D}$."

Second we replace the limiting processes leading to the integral by processes analogous to those introduced by Birkhoff [2, pp. 50-52] and used by Phillips [1] and Rickart [1]. Thus we say that $\left.{ }^{*}\right) \int \varsigma \mu\{t, d t\}=x$ if given any neighborhood $U(x)$ of $x$ there is an L-P: $L$ with the property that if $L^{\prime}: \sigma_{i}^{a}<L$, then there are finite sets $A_{i}$ such that if $B_{i}$ are finite sets containing $A_{i}$, then $\prod_{i=1}^{n} \prod_{b \in B_{i}} \mu\left\{t_{i}^{a}, \sigma_{i}^{a}\right\} \subset \mathcal{U}(x)$.

With these changes it is easy to find determinations of $\mathfrak{D}$ and $\mu$ so that $\left({ }^{*}\right) \int_{\varsigma} \mu\{t, d t\}$ is $(1)$ the $U$-integral of Phillips $[1$, p. 118] or (2) the $\mathcal{V}$-integral of Rickart [1, p. 502].

The analysis of $\S 8$ applies without modification to yield the usual existence theorem for Riemann-Stieltjes integrals. Let $\mathfrak{D}$ be the additive group of real numbers (or more generally any Banach space) and let numerically-valued functions $f$ and $\phi$ with domain $[\alpha, \beta]$ be given. (More generally $f$ may have its values in the Banach space $\mathfrak{D}$.) Now let $\mu\{t, s\}$ be defined as

$$
f(t) \sum_{i=1}^{n}\left[\phi\left(t_{i}^{2}\right)-\phi\left(t_{i}^{1}\right)\right]
$$

whenever $s$ is a union of intervals with left- and right-hand end points $t_{t}^{1}, t_{t}^{2}$ respectively. A differential of this form will be called a Stieltjes differential.

If $\mu$ is the Stieltjes differential

$$
\mu\{t, s\}=f(t) \cdot \sum_{i=1}^{n}\left[\phi\left(t_{i}^{2}\right)-\phi\left(t_{i}^{1}\right)\right]
$$

the following are readily verified.

(11.11) If $f$ is bounded and $\phi$ is of bounded variation on $[\alpha, \beta]$, then $\mu$ is of bounded $R$-variation on $[\alpha, \beta]$.

(11.12) If $\phi$ is of bounded variation and $m(s)$ is the (total) variation of $\phi$ on $s$ and $f$ is continuous at $t_{0}$, then $\operatorname{Osc}_{m}\left(\mu \mid t_{0}\right)=0$.

(11.13) $\mu$ is approximately multiplicative.

(11.14) ThEOREM. If $\mu$ is the Stieltjes differential $\mu\{t, s\}=f(t)$ . $\sum_{i=1}^{n}\left[\phi\left(t_{i}^{2}\right)-\phi\left(t_{i}^{1}\right)\right]$, then $\int_{\alpha}^{\beta} \mu\{t, d t\}$ is the Riemann-Stieltjes integral $\int_{\alpha}^{\beta} f(t) d \phi(t)$.

Proof. With the Convention 6.1, $\int_{\alpha}^{\beta} f(t) d \phi(t)$ is just $* \int_{\alpha}^{\beta} \mu\{t, d t\}$. For any S-P: $\Pi: \Delta_{i}$ and any element, $x=\sum_{i=1}^{n} f\left(t_{i}^{2}\right)\left[\phi\left(t_{i}\right)-\phi\left(t_{i}^{1}\right)\right]$, of $J^{*}(\mu, \Pi)$ we can obtain, by uniting those $\Delta_{i}$ for which the $t_{i}$ coincide and removing end points where necessary, a $\Pi^{\prime}$ with $\left|\Pi^{\prime}\right| \leqq 2|\Pi|$ such that $x \in J\left(\mu, \Pi^{\prime}\right)$. Hence $* \int_{\alpha}^{\beta} \mu\{t, d t\}=\int_{\alpha}^{\beta} \mu\{t, d t\}$.

(11.15) Theorem. If $\phi$ is of bounded variation and if $f$ is bounded and is 
continuous except on a set $E$ such that the variation of $\phi$ on $E$ is zero, then the Riemann-Stieltjes integral $\int_{\alpha}^{\beta} f(t) d \phi(t)$ exists.

Proof. Let $\mu$ be the Stieltjes-differential $\mu\{t, s\}=f(t) \sum_{i=1}^{n}\left[\phi\left(t_{t}^{2}\right)-\phi\left(t_{i}^{1}\right)\right]$. In view of Theorems 11.14 and 5.20 we need only show that $(R) \int_{\alpha}^{\beta} \mu\{t, d t\}$ exists. From (3.23), (11.11), 11.12, and (11.13) we infer that all the hypotheses of Theorem 8.1 are satisfied except possibly the condition $\int_{\alpha}^{\beta} F_{m, \xi_{0}}(t) d m<+\infty$. But from Definition 4.5 it is easily seen that $\operatorname{Osc}_{m}(\mu \mid \Delta)$ is less than or equal to the bound of $f$, from which the desired result follows immediately.

12. Product integrals. The following paragraphs are to be read in conjunction with a paper by Masani [1], references to which are preceded by an $[\mathrm{M}]$.

Let us say that $\mu$ is a product differential if $\mathfrak{D}$ is a normed ring and $\mu\{t, \sigma\}$ $=1+F(t) \cdot|\sigma|$, where $F$ is a function on $[\alpha, \beta]$ with values in $\mathfrak{D}$. We have already seen that if $\mu$ is a product differential then: $\mathfrak{D}$ is relatively stable $(3.24) ; \mu$ is bounded if and only if $F$ is bounded $(4.6) ; \operatorname{Osc}(\mu \mid t)=0$ if and only if $F$ is continuous at $t(4.7)$. The following is a consequence of equation [M] (4.32).

(12.1) If $\mu$ is the product differential $\mu\{t, \sigma\}=1+F(t) \cdot|\sigma|$ and $F$ is bounded, then $\mu$ is approximately multiplicative.

(12.2) THEOREM. If $\mu$ is the product differential $1+F(t) \cdot|\sigma|$, then $(S) \int_{\alpha}^{\beta} \mu\{t, d t\}$ coincides with the product integral $\int_{\alpha}^{-\beta}(1+F d t)$ defined by Masani (Definition [M] 6.2).

Proof. With trivial modifications, the proof of [M] Theorem 11.5 can be used to show that if $(S) \int_{\alpha}^{\beta} \mu\{t, d t\}$ exists, then $F$ is bounded.

By Theorem 6.12 the existence of $(S) \int_{\alpha}^{\beta} \mu\{t, d t\}$ implies the existence of $\lim _{|\mathrm{II}| \rightarrow 0} S J^{*}(\mu, \Pi)$. Comparing Definitions 3.17 and [M] (9.1) and using the Convention 6.1, we see that $S \frown(F, \Pi) \subset S J^{*}(\mu, \Pi)$ whenever $\Pi$ is an S-P*. Accordingly $\lim _{|\Pi| \rightarrow 0} S \frown(F, \Pi)=\lim _{|\Pi| \rightarrow 0} S J^{*}(\mu, \Pi)=(S) \int_{\alpha}^{\beta} \mu\{t, d t\}$. By $[\mathrm{M}]$ (8.2) and Definitions [M] (6.1) and [M] (9.1), $\int_{\alpha}^{\beta}(1+F d t)$ exists and is (S) $\int_{\alpha \mu}^{\beta} \mu\{t, d t\}$.

On the other hand, if $\int_{\alpha}^{\beta}(1+F d t)$ exists, then $F$ and hence $\mu$ is bounded ([M] Theorem 11.5). Comparing Definitions 3.2 and [M] (9.1), we see that $J(\mu, \Pi) \subset S \frown(F, \bar{\Pi})$, where $\bar{\Pi}$ is the $\mathrm{S}^{-\mathrm{P}^{*}}$ whose intervals are the closures of those of $\Pi$. From Definitions [M] (5.2), [M] (6.1) and [M] (9.1) we see that if $\int_{\alpha}^{\beta}(1+F d t)$ exists, then so does $\lim _{\Pi \downarrow} S(F, \overline{\mathrm{II}})$ and that the two are equal. Hence $\lim _{\Pi \downarrow} J(\mu, \Pi)=\int_{\alpha}^{\beta}(1+F d t)$ and, from Theorem $5.8, \int_{\alpha}^{\beta}(1+F d t)$ $=(S) \int_{\alpha}^{\beta} \mu\{t, d t\}$.

(12.3) THEOREM. If $\mu$ is a product differential, then $S$ and $R$ integration are equivalent. 
Proof. From Theorem 5.6 we know that $R$-integrability implies $S$-integrability to the same integral.

Assume that the product differential $\mu\{t, \sigma\}=1+F(t)|\sigma|$ is $S$-integrable to $x$. From the proof of the preceding theorem $F$ has a bound $B$. Now let $\epsilon>0$ be given. We can find a $\delta_{1}>0$ so that $|\Pi|<\delta_{1}$ and $y \in S J(\mu, \Pi)$ imply $\rho(y, x)<\epsilon$. Let $\Pi: \Delta_{i}$ be any S-P with $|\Pi|<\delta_{1}$ and let $z$ be any element of $R J(\mu, \Pi)$. Then

$$
z=\prod_{i=1}^{n} \prod_{j=1}^{n_{i}}\left(1+F\left(t_{i}^{j}\right)\left|s_{i}^{j}\right|\right)
$$

where $t_{i}^{j} \in s_{i}^{j}$ and $U_{j=1}^{n_{i}} s_{i}=\Delta_{i}^{j}$. Let $z^{\prime}=\prod_{i=1}^{n}\left(1+\sum_{j=1}^{n_{i}} F\left(t_{i}^{j}\right)\left|s_{i}^{j}\right|\right)$. From equations [M] (4.22), [M] (4.32), and [M] (4.42) we infer

$$
\rho\left(z, z^{\prime}\right) \leqq B(\beta-\alpha) \exp B(\beta-\alpha)[\exp B|\Pi|-1]
$$

Now

$$
\begin{aligned}
z^{\prime} & =\prod_{i=1}^{n}\left\{\sum_{j=1}^{n_{i}} \frac{\left|s_{i}^{j}\right|}{\left|\Delta_{i}\right|}\left[I+\left|\Delta_{i}\right| \cdot F\left(t_{i}^{j}\right)\right]\right\} \\
& =\sum\left\{\left(\prod_{i=1}^{n} \frac{\left|s_{i}^{j(i)}\right|}{\left|\Delta_{i}\right|}\right) \prod_{i=1}^{n}\left[I+\left|\Delta_{i}\right| \cdot F\left(t_{i}^{j(i)}\right)\right]\right\}
\end{aligned}
$$

where the sum is to be taken over all functions $j$ with domain $1,2, \cdots, n$ such that $1 \leqq j(i) \leqq n_{i}$. Since the sum of the coefficients $\left(\prod_{i=1}^{n}\left|s_{i}^{j(i)}\right| /\left|\Delta_{i}\right|\right)$ is 1 and none of them is negative, it follows that $\rho\left(x, z^{\prime}\right)=\left|x-z^{\prime}\right| \leqq \sup _{j(i)} \mid x$ $-\prod_{i=1}^{n}\left[I+\left|\Delta_{i}\right| F\left(t_{t}^{(t)}\right)\right] \mid$. As each of these products is in $S J(\mu, \Pi)$, this yields $\rho\left(x, z^{\prime}\right)<\epsilon$. Combining this 'with (12.4) we see that if $|\Pi|<\delta_{1}$ and $|\Pi|<\delta_{2}=(1 / B) \log (1+\epsilon \exp [B(\beta-\alpha)] /[B(\beta-\alpha)])$, then any element of $R J(\mu, \mathrm{II})$ is within $2 \epsilon$ of $x$, that is, $(R) \int_{\alpha}^{\beta} \mu\{t, d t\}=x$.

Masani's product integral includes the Riemann product integrals of matrix-valued functions studied by Volterra [1, 2, 3], Schlesinger [1], Rasch [1], and Volterra and Hostinsky [1], but not the Lebesgue integral defined by Schlesinger [1, pp. 54-57].

(12.5) Theorem. If $\mu$ is the product differential $\mu\{t, \sigma\}=1+F(t) \cdot|\sigma|$ and Schlesinger's Lebesgue product integral $\int_{\alpha}^{-\beta}(I+F d t)$ exists, then so does (L) $\int_{\alpha}^{\beta} \mu\{t, d t\}$, and the two are equal.

Proof. Schlesinger's $[F]=n \max \left|F_{i j}\right|$ [Schlesinger 1, p. 35] does not satisfy Gelfand's axiom $\delta^{\prime}$ [Gelfand 1, p. 3] for a normed ring, but Gelfand's construction [op. cit. pp. 3-4] leads to a norm $|F|$ satisfying the relation $[F] / n \leqq|F| \leqq[F]$. For convenience we shall use $|F|$ rather than $[F]$. This does not change Schlesinger's definitions essentially. 
A bounded matrix-valued function $F$ is integrable in the sense of Schlesinger if there is a sequence, $F_{1}, F_{2}, \cdots$, of step functions converging almost everywhere to $F$. Without loss of generality we may assume the $F_{i}$ to be uniformly bounded.

Let $\epsilon>0$ be given and let $B$ be a bound for $F, F_{1}, F_{2}, \ldots$ By (3.24) and Definition 3.22 there is a $\delta=\delta_{B(\beta-\alpha)}(\epsilon)$ such that $\rho\left(x_{i}, 1\right)<B(\beta-\alpha)$ and $\sum_{i=1}^{n} \rho\left(x_{i}, y_{i}\right)<\delta$ imply $\rho\left(\prod_{i=1}^{n} x_{i}, \prod_{i=1}^{n} y_{i}\right)<\epsilon$. By Egoroff's theorem [Saks 1, p. 18] there is an L-P $L^{\prime}: \sigma_{i}, i=0,1$, and an integer $N$ such that $\left|\sigma_{0}\right|<\delta / 4 B$ and $\nu>N, t \in \sigma_{1}$ imply $\left|F(t)-F_{\nu}(t)\right|<\delta / 2(\beta-\alpha)$. Let $\mu_{\nu}\{t, \sigma\}=1+F_{\nu}(t) \cdot|\sigma|$. By Theorems 8.9, 10.1, 9.4, 9.7, and 9.8 there is an L-P, $L_{v}$, such that $d\left(L J\left(\mu_{\nu}, L_{\nu}\right)\right)<\epsilon$. Let $L_{\nu}^{*}=L_{\nu} \cdot L^{\prime}$ and let

$$
y=\prod_{i=1}^{n} \prod_{a \in A_{i}}\left(1+F\left(t_{i}^{a}\right)\left|\sigma_{i}^{a}\right|\right)
$$

be any element of $L J\left(\mu, L_{v}^{*}\right)$. Then

$$
z=\prod_{i=1}^{n} \prod_{a \in A_{i}}\left(1+F_{\nu}\left(t_{i}^{a}\right) \cdot\left|\sigma_{i}^{a}\right|\right)
$$

is an element of $L J\left(\mu_{\nu}, L_{\nu}^{*}\right)$. By Definition 9.1 there are finite sets $B_{i}$ such that if $y^{\prime}=\prod_{i=1}^{n} \prod_{a \in B_{i}}\left(1+F\left(t_{i}^{a}\right) \cdot\left|\sigma_{i}^{a}\right|\right)$ and $z^{\prime}=\prod_{i=1}^{n} \prod_{a \in B_{i}}\left(1+F_{\nu}\left(t_{i}^{a}\right)\left|\sigma_{i}^{a}\right|\right)$, then $\rho\left(y^{\prime}, y\right)<\epsilon$ and $\rho\left(z^{\prime}, z\right)<\epsilon$. Now

$$
\begin{aligned}
& \sum_{i=1}^{n} \sum_{b \in B_{i}} \rho\left(1+F\left(t_{i}^{a}\right)\left|\sigma_{i}^{a}\right|, 1+F_{\nu}\left(t_{i}^{a}\right)\left|\sigma_{i}^{a}\right|\right) \\
& \quad=\sum_{\sigma_{i}^{a} \subseteq \sigma_{0}}\left|F\left(t_{i}^{a}\right)-F_{\nu}\left(t_{i}^{a}\right)\right| \cdot\left|\sigma_{i}^{a}\right|+\sum_{\sigma_{i}^{a} \subset \sigma_{1}}\left|F\left(t_{i}^{a}\right)-F_{\nu}\left(t_{i}^{a}\right)\right| \cdot\left|\sigma_{i}^{a}\right| \\
&<2 B\left|\sigma_{0}\right|+\frac{\delta}{2(\beta-\alpha)}\left|\sigma_{1}\right| \\
&<\delta .
\end{aligned}
$$

By Definition 3.22, $\rho\left(y^{\prime}, z^{\prime}\right)<\epsilon$. Thus any element of $L J\left(\mu, L_{\nu}^{*}\right)$ is within $3 \epsilon$ of an element of $L J\left(\mu_{\nu}, L_{v}\right)$. Since the diameter of the latter is less than $\epsilon$, the diameter of the former is less than $7 \epsilon$ and, by Theorem 9.4, $(L) \int_{\alpha}^{\beta} \mu\{t, d t\}$ exists. Obviously also $(L) \int_{\alpha}^{\beta} \mu\{t, d t\}=\lim _{n \rightarrow \infty} \lim _{L \downarrow} L J\left(\mu_{\nu}, L\right)=\int_{\alpha}^{\beta}(1+F d t)$.

For unbounded functions, $F$, Schlesinger's definition is not altogether clear unless $|F|$ is summable. In this case it is easy to prove that

$$
\begin{aligned}
\int_{\alpha}^{\frown^{\beta}}(1+F d t) & =\lim _{N \rightarrow \infty}(L) \int_{\alpha}^{\beta} \mu_{N}\{t, d t\} \\
& =(L) \int_{\alpha}^{\beta} \mu\{t, d t\}
\end{aligned}
$$


where

$$
\mu_{N}\{t, \sigma\}=\left\{\begin{array}{lll}
1+F(t)|\sigma| & \text { if } & |F(t)| \leqq N \\
1 & \text { if } & |F(t)|>N .
\end{array}\right.
$$

Since the ring of matrices is finite-dimensional, every Riemann integrable function is continuous almost everywhere and hence integrable in the sense of Schlesinger. An analogue of Graves' example [Graves 1, p. 166] shows that this is not the case when the Schlesinger definition is extended to arbitrary normed rings.

Our methods also enable us to infer the existence of product integrals analogous to Riemann-Stieltjes integrals. Let $\mathfrak{D}$ be a normed ring and let functions $F$ and $\Phi$ with domain $[\alpha, \beta]$ and range in $\mathfrak{D}$ be given. Let $\mu\{t, s\}$ $=1+F(t) .\left[\sum_{i=1}^{n}\left(\Phi\left(t_{i}^{2}\right)-\Phi\left(t_{i}^{1}\right)\right)\right]$ where $t_{i}^{2}$ and $t_{i}^{1}$ are the right-and left-hand end points of the intervals forming $s$. Such a differential $\mu$ will be called a Stieltjes product differential.

(12.6) Definition. If $\Phi$ is a function with domain $[\alpha, \beta]$ and range in a normed ring $\mathfrak{D}$, the variation, $V(\Phi, \Delta)$, of $\Phi$ over an interval $\Delta \subset[\alpha, \beta]$ is $\sup \sum_{i=1}^{n}\left|\Phi\left(t_{i}^{2}\right)-\Phi\left(t_{i}^{1}\right)\right|$ where the supremum is taken over all choices of $t_{1}^{1}, t_{1}^{2} \in \Delta$ such that $t_{1}^{1}<t_{1}^{2} \leqq t_{2}^{1}<t_{2}^{2} \leqq \cdots \leqq t_{n}^{1}<t_{n}^{2}$.

If $V(\Phi,[\alpha, \beta])$ is finite, $V(\Phi, \Delta)$ is an additive function of an interval and of bounded variation. Accordingly it [Saks 1, pp. 64, 65] determines a measure $m_{\Phi}(\sigma)$ defined at least for all Borel sets $\sigma$.

If $\mu$ is the Stieltjes product differential $\mu\{t, s\}=1+F(t)\left[\sum_{i=1}^{n}\left\{\Phi\left(t_{i}^{2}\right)\right.\right.$ $\left.\left.-\Phi\left(t_{8}^{1}\right)\right\}\right]$, direct calculation shows:

(12.7) If $F$ is continuous at $t_{0}$, then $\operatorname{Osc}_{m_{\Phi}}\left(\mu, t_{0}\right)=0$;

(12.8) If $F$ is bounded and $V(\Phi,[\alpha, \beta])<+\infty$, then $\mu$ is of bounded variation.

However a Stieltjes product differential is not in general approximately multiplicative and Theorem (8.8) is not immediately applicable. In the proof of that theorem approximate multiplicativity was used only in establishing (8.2), which we now derive otherwise.

If $F$ and $\Phi$ are given, let

$$
M(s)=\sum_{i=1}^{n}\left(\Phi\left(t_{i}^{2}\right)-\Phi\left(t_{i}^{1}\right)\right)
$$

where $t_{t}^{2}$ and $t_{t}^{1}$ are the right- and left-hand end points of the intervals forming s. We must show that there is a $\delta<0$ such that $|\Pi|<\delta$ implies (8.2), that is,

$$
\sum_{i=1}^{n}\left|1+F(t) M\left(\Delta_{i}\right)-\prod_{j=1}^{n_{i}}\left(1+F(t) M\left(s_{i}^{j}\right)\right)\right|<\delta_{V}(\epsilon) .
$$

If $B$ is a bound for $F$, then straightforward calculation shows that 


$$
\begin{aligned}
\left|1+F(t) M\left(\Delta_{i}\right)-\prod_{j=1}^{n_{i}}\left(1+F(t) \cdot M\left(s_{i}^{j}\right)\right)\right| \leqq B^{2} m_{\Phi}\left(\Delta_{i}\right) \cdot m_{\Phi}\left(\underset{j \neq j_{0}}{U} s_{i}^{j}\right) & \\
& +B m_{\Phi}\left(\Delta_{i}\right)\left(1+B m_{\Phi}\left(\Delta_{i}\right)\right)\left(\exp \left[B \cdot m_{\Phi}\left(\bigcup_{j \neq j_{0}} s_{i}^{j}\right)\right]-1\right)
\end{aligned}
$$

for any $j_{0}, 1 \leqq j_{0} \leqq n_{i}$. The desired result follows if the right-hand side can be made less than $\delta_{V}(\epsilon) m_{\Phi}\left(\Delta_{i}\right) / m_{\Phi}([\alpha, \beta])$. This is the case if we can make $m_{\Phi}\left(\bigcup_{j \neq j_{0}} s_{i}^{\jmath}\right)$ arbitrarily small uniformly in $i$.

Now $\phi(t)=m_{\Phi}([\alpha, t])$ is a nondecreasing upper semi-continuous function on $\alpha \leqq t \leqq \beta$ and hence $m_{\Phi}([\alpha, t])=f(t)+g(t)$ where $f$ is a continuous nondecreasing function and $g$ is of the form $g(t)=\sum_{\xi_{k} \leqq t} s\left(\xi_{k}\right)$ where $s\left(\xi_{k}\right)$ is the saltus, $\phi\left(\xi_{k}+0\right)-\phi\left(\xi_{k}-0\right)$, of $\phi$ at $\xi_{k}$. If $\epsilon>0$ is given, we can find $\delta_{1}$ so that $\left|t_{2}-t_{1}\right|<\delta_{1}$ implies $\left|f\left(t_{2}\right)-f\left(t_{1}\right)\right|<\epsilon / 2$. We can also find $N$ so that $\sum_{k=N+1}^{\infty} s\left(\xi_{k}\right)<\epsilon / 2$. Let $\delta_{2}=2^{-1} \inf _{i, j \leqq N}\left|\xi_{i}-\xi_{j}\right|$. Now let $\delta$ be the smaller of $\delta_{1}$ and $\delta_{2}$ (or, if $N=0$, just $\delta_{1}$ ). If $\left|\Pi: \Delta_{i}\right|<\delta$ and $R: s_{i}^{j}<\Pi$, then no $\Delta_{i}$ can contain more than one $\xi_{k}$ with $k \leqq N$. If $\Delta_{i}$ does contain such a $\xi_{i}$, let $s_{i}^{30}$ be the $s_{i}^{j}$ containing it. Now $m_{\Phi}\left(s_{i}^{j}\right)=\sum_{l}\left(f\left(r_{l}\right)-f\left(t_{l}\right)+g\left(r_{l}\right)-g\left(t_{l}\right)\right)$ where the $r$ 's and $t$ 's are the right and left end points of the intervals forming $s_{i}^{3}$. Thus $m_{\Phi}\left(U_{j \neq j_{0}} s_{i}^{j}\right) \leqq f(r)-f(t)+\sum_{\xi_{i} \in \Delta_{i}, i>N} s\left(\xi_{i}\right)<\epsilon$ where $r$ and $t$ are the end points of $\Delta_{i}$.

This completes the proof that (8.2) is valid for sufficiently small $|\Pi|$ whenever $\mu$ is the product differential $\mu\{t, s\}=F(t) \cdot \sum_{i=1}^{n}\left(\Phi\left(r_{i}\right)-\Phi\left(t_{i}\right)\right)$ with $F$ bounded and $\Phi$ of bounded variation on $[\alpha, \beta]$.

The remainder of the proof of Theorem (8.1) is valid under these conditions. We have thus proved:

(12.10) THEOREM. If $\Phi$ and $F$ are functions from $[\alpha, \beta]$ to a normed ring and if $\Phi$ is of bounded variation and if $F$ is continuous except possibly on a set $E$ where $\Phi$ has zero variation, then the Riemann-Stieltjes product integral

$$
\text { (R) } \int_{\alpha}^{\beta}[1+F(t) d \Phi(t)]=\lim _{|\mathrm{II}| \rightarrow 0} \prod_{i=1}^{n} \prod_{j=1}^{n}\left[1+F\left(t_{i}^{j}\right) M\left(s_{i}^{j}\right)\right]
$$

exists where $t_{i}^{j} \in s_{i}^{\prime}, R: s_{i}^{\prime}<\Pi$, and $M\left(s_{i}^{j}\right)$ is defined by (12.9).

We may, by Theorem 5.20, replace the $R$-integration in Theorem 12.10 by $S$-integration. It is also desirable, to conform to the usual practice in additive integration, to be able to replace $S$-integration by $S^{*}$-integration. Theorem 6.2 does not apply, but like Theorem 8.8 its proof may be modified to cover the present situation. We thus have:

(12.11) ThEOREM. Under the hypotheses of Theorem (12.10) the integral

$$
\left(S^{*}\right) \int_{\alpha}^{\beta}[1+F(t) d \Phi(t)]=\lim _{|\overline{\mathbf{n}}| \rightarrow 0} \prod_{i=1}^{n}\left[1+F\left(t_{i}\right)\left(\Phi\left(t_{i}^{2}\right)-\Phi\left(t_{i}^{1}\right)\right]\right.
$$


exists where $\bar{\Pi}: \Delta_{i}$ is any partition of $[\alpha, \beta]$ into nonoverlapping intervals, $t_{i} \in \Delta_{i}$, and $t_{i}^{2}$ and $t_{i}^{1}$ are the right-and left-hand end points of $\Delta_{i}$.

In the following paragraphs references preceded by $\left[\mathrm{B}^{\prime}\right]$ are to a paper [Birkhoff 3] in which Birkhoff studies the product integrals of nonlinear transformations of a Banach space $B$ into itself.

Following Birkhoff we shall use $p, q$, and so on for elements of $B ;|p|$ for the norm of $p \in B$; italic capitals for transformations of $B$ into itself; 0 for the identity transformation, $p X$ for the image of $p$ under the transformation $X$. and $|X|$ and $\|X\|$ for the $D$ - and $L$-moduli of $X$ ([B'] p. 112).

Let $D$ be the set of all $X$ such that $|X|<+\infty$ and $\|X\|<+\infty$. The binary operation assumed in $\mathfrak{D}$ is defined by $p(X \cdot Y)=(p X) Y$. Addition, subtraction, and scalar multiplication defined by $\left[\mathrm{B}^{\prime}\right](13),\left[\mathrm{B}^{\prime}\right] 14$ will be used where convenient. $\mathfrak{D}$ is metrized by the metric $\rho(X, Y)=|X-Y|$.

$\mathfrak{D}$ is neither complete in its metric, nor relatively stable. However from [B'] pp. 113, 144 we obtain:

(12.12) For any $M$ the set of all $X \in D$ such that $\|X\| \leqq M$ is complete.

(12.13) Given $\epsilon, t>0$ there is a $\delta_{L}(\epsilon)$ such that

$$
\sum_{i=1}^{n}\left\|X_{i}\right\|<L, \quad \sum_{i=1}^{n}\left|X_{i}-Y_{i}\right|<\delta
$$

imply

$$
\left|\prod_{i=1}^{n} X_{i}-\prod_{i=1}^{n} Y_{i}\right|<\epsilon
$$

A differential $\mu$, such that $\mu\{t, \sigma\}=X(t) \cdot|\sigma|$ where $X$ is a function with domain $[\alpha, \beta]$ and range in $\mathfrak{D}$, will be referred to as a Birkhoff differential. The following properties of Birkhoff differentials, $\mu\{t, \sigma\}=X(t) \cdot|\sigma|$, are readily verified by inspecting the references listed after each statement.

(12.14) If $|X(t)|$ is bounded, $\mu$ is bounded.

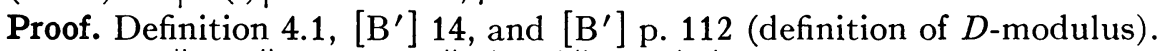
(12.15) If $\|X(t)\| \leqq B$, then $\|\mu\{t, \sigma\}\| \leqq B \cdot|\sigma|$.

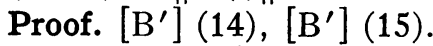

(12.16) Osc $\left(\mu \mid t_{0}\right)=0$ if and only if $X$ is continuous (in the metric $\rho$ ) at $t_{0}$.

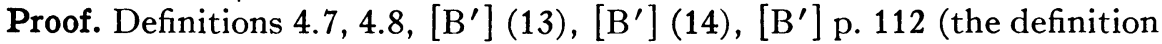
of $D$-modulus).

(12.17) If $|X(t)|$ and $\|X(t)\|$ are bounded, then $\mu$ is approximately multiplicative.

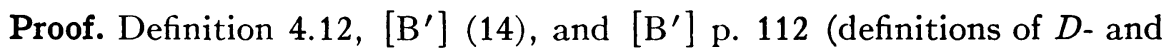
$L$-moduli).

Now Birkhoff's Riemann integral $\int_{\alpha}^{\beta} X(t) d t\left(\left[B^{\prime}\right] \S 6\right.$, the $B(A)$ in equation $\left[\mathrm{B}^{\prime}\right]$ (11) does not appear in the later discussion) is clearly identical with the integral $\left(S^{*}\right) \int_{\alpha}^{\beta} \mu\{t, d t\}$ of our Theorem 6.2 where $\mu\{t, \sigma\}=X(t) \cdot|\sigma|$. In the 
proof of Theorem 6.2 completeness plays no role, while relative stability is used only to infer equation (6.10) from (6.11). If $\|X(t)\| \leqq B$, we obtain $\sum_{i=1}^{n}\left\|\mu\left\{t_{i}, \Delta_{i}\right\}\right\| \leqq B(\beta-\alpha)$ from (12.15), and (6.10) can be obtained from (12.13) instead of Definition 3.22. Accordingly we obtain:

(12.18) THEOREM. If $\mu$ is the Birkhoff differential $\mu\{t, \sigma\}=X(t) \cdot|\sigma|$, then $\int_{\alpha}^{\beta} X(t) d t=Y$ implies $(S) \int_{\alpha}^{\beta} \mu\{t, d t\}=Y$. If $|X(t)|$ and $\|X(t)\|$ are bounded, the converse is true.

In proving Theorem 8.8 we used (5.5), which depends on completeness. However it would be sufficient to know that all the products considered lie in a complete subset of $\mathfrak{D}$. If $\|X(t)\|<B$, this is ensured by $\left[B^{\prime}\right]\left(16^{\prime}\right)$. Also relative stability may be replaced by (12.13) provided $\|X(t)\|<B$. Accordingly we can infer that if $|X(t)|$ and $\|X(t)\|$ are bounded, the Birkhoff differential $\mu\{t, \sigma\}=X(t) \cdot|\sigma|$ is $R$-integrable provided $X(t)$ is continuous almost everywhere in the metric $\rho$. In view of Theorems 5.20 and 12.18 this extends Birkhoff's theorem [ $\left.\mathrm{B}^{\prime}\right]$ (4) on Riemann integrability to discontinuous functions.

(12.19) Theorem. If $|X(t)|$ and $\|X(t)\|$ are bounded and if $X(t)$ is continuous in the metric $\rho(X, Y)=|X-Y|$ for almost all $t$ on a finite interval $[\alpha, \beta]$, then $X(t)$ is R-integrable on $[\alpha, \beta]$.

\section{GARRETT BirkHoFF}

\section{BIBLIOGRAPHY}

1. Integration of functions with values in a Banach space, Trans. Amer. Math. Soc. vol. 38 (1935) pp. 357-378.

2. Moore-Smith convergence in general topology, Ann. of Math. (2) vol. 38 (1937) pp. 39-56.

3. On product integration, Journal of Mathematics and Physics (Massachusetts Institute of Technology) vol. 16 (1937) pp. 104-132.

I. GELFAND

1. Normierte Ringe, Rec. Math. (Mat. Sbornik) N.S. vol. 9 (1941) pp. 3-24.

Lawrence M. Graves

1. Riemann integration and Taylor's theorem in general analysis, Trans. Amer. Math. Soc. vol. 29 (1927) pp. 163-177.

F. HAUSDORFF

1. Mengenlehre, 2d ed., Berlin and Leipzig, de Gruyter, 1927.

H. LEBESGUE

1. Intégrale, longueur, aire, Annali di Matematica (3) vol. 7 (1902) pp. 231-359.

P. R. MASANI

1. Multiplicative Riemann integration in normed rings, Trans. Amer. Math. Soc. vol. 61 (1947) pp. 147-192.

E. H. Moore and H. L. Smith

1. A general theory of limits, Amer. J. Math. vol. 44 (1922) pp. 102-121.

R. S. Phillips

1. Integration in a convex linear topological space, Trans. Amer. Math. Soc. vol. 47 (1940) pp. 114-145.

G. Baley Price

1. The theory of integration, Trans. Amer. Math. Soc. vol. 47 (1940) pp. 1-50. 
G. RASCH

1. Zur Theorie und Anwendung des Productintegrals, J. Reine Angew. Math. vol. 171 (1934) pp. 65-119.

C. E. RICKART

1. Integration in a convex linear topological space, Trans. Amer. Math. Soc. vol. 52 (1942) pp. 498-521.

B. RIEMANN

1. Ueber die Darstellbarkeit einer Function durch eine trigonometrische Reihe, Riemann's Gesammelte Werke, Leipzig, Teubner, 1876, pp. 213-250.

StanisLaw Saks

1. Theory of the integral, Monografje Matematyczne, vol. 7, Warsaw and Lw6w, 1937.

L. SCHLESINGER

1. Neue Grundlagen für einen Infinitesimalkalkul der Matrizen, Math. Zeit. vol. 33 (1931) pp. 33-61.

2. Weitere Beiträge zum Infinitesimalkalkul der Matrizen, Math. Zeit. vol. 35 (1932) pp. 485-501.

T. J. Stieltjes

1. Recherches sur les fractions continues, Oeuvres Complètes de Stieltjes, Groningen, Noordhoff, 1918, vol. 2, pp. 402-566.

V. VOLTERrA

1. Sulle equazioni differenziali lineari, Rendiconti Accademia dei Lincei (4) vol. 3, sem. 1 (1887) pp. 393-396.

2. 3. Sui fondamenti della teoria delle equazioni differenziali, Memorie Sociètà Italiana delle Scienze (3) vol. 6 (1887); (3) vol. 12 (1902) pp. 3-68.

V. VolterRa AND B. Hostinsky

1. Operations infinitésimal linéaires, Paris, Gauthier-Villars, 1938.

BROWN UNIVERSITY,

Providence, R. I. 